\title{
Regeneration sekundärer Sexualcharaktere bei den Amphibien.
}

\author{
Von \\ Dr. phil. Paul Kammerer. \\ (Aus der Biologischen Versuchsanstalt in Wien.) \\ Mit Tafel II und III. \\ Eingegangen am 31. August 1907.
}

\section{Inhaltsübersicht.}

I. Ziel und Geschichte der Versuche

II. Darstellung der Versuche . . . . . . . . . . . . . . . . . . . 80

A. Anuren . . . . . . . . . . . . . . . 85

1. Brunftschwielen der Männchen . . . . . . . . . . . . $8 \tilde{5}$

a. Zehenschwielen am Vorderbein von Bufo viridis . . . . . 85

b. Zehen- und Armschwielen von Bombinator pachypus . . . 86

2. Schallblasen der Männchen . . . . . . . . . . . . 88

a. Kehlsack von Hyla arborea . . . . . . . . . . . . . . . 88

b. Schallblasen von Ranc escallenta . . . . . . . . . . . . . 91

B. Urodelen . . . . . . . . . . . . . . . . . . . . . . . . . . 93

1. Kïmme der männlichen Wassermolche. . . . . . . . . . . 93

a. Ganzrandige Kämme (Triton alpestris, marmoratus, blasi $i_{\text {) } 93}$

b. Gezackte Kämme (Triton cristatics und vulgaris . . . . . . 100

2. Andre Hantwucherungen . . . . . . . . . . . . . . . 106

a. Hautsaum an der Oberlippe von Triton cristatus. . . . . . 106

b. Zehenlappen von Triton vulgaris . . . . . . . . . . . . 107

e. Schwimmhäute von Triton palmatus . . . . . . . . . . 108

d. Endfaiden am Schwanze mehrerer Tritonarten . . . . . . . 109

e. Halswarze von Triton pyrrhogaster . . . . . . . . . 111

3. Sporn von Triton rusconii . . . . . . . . . . . . . . . . 111

4. Farbcharaktere von Triton cristatus . . . . . . . . . . . 114

a. Schwanzstreifen des Männchens . . . . . . . . . . . 114

b. Rückenlängslinie des Weibchens . . . . . . . . . . 115

III. Zusammenfassung . . . . . . . . . . . . . . . . . . 116

A. Regenerationen. . . . . . . . . . . . . . . 116

B. Kompensationen . . . . . . . . . . . . . . . . . . . . . 120

C. Andre Ergebnisse . . . . . . . . . . . . . . . . . . . . . . . 122

IV. Literaturverzeichnis . . . . . . . . . . . . . . . . . . . . 122

V. Erklärung der Abbildungen . . . . . . . . . . . . . 124 


\section{Ziel und Geschichte der Versuche.}

Die Tatsache, daß viele regenerierbare morphologische Einheiten entweder vorläufig oder definitiv nicht in derjenigen Gestaltung wiederkehren, welche sie frihher, bevor ihr Verlust erfolgt war, am Körper des betroffenen Tieres aufwiesen, sondern ontogenetisch, in manchen Fällen phylogenetisch tieferstehenden Gebilden ähneln, bat eines der interessantesten Probleme in der Regenerationslebre erstehen lassen. Seiner Lösung näber zu treten, scheint gleichbedeutend zu sein mit einer Verheißung, in so beschaffene Fragen der Systematik, der Entwicklungs- und Abstammungslehre, daß ihnen auf andre Weise schwer beizukommen ist, helles Licht $\mathrm{zu}$ werfen.

Vorläufig freilich ist der Stand des bezeichneten Problems noch zu sehr geeignet, einen scharfen Gegensatz der Meinungen hervorzurufen, ähnlich wie er auch noch besteht betreffend die Auffassung der Regeneration als Anpassungs- oder als allgemeine Erscheinung der Naturkörper. Also was dort die einen (unter ihnen BarfurTh, Bordage, Giard, E. Schultz, Weismann, Werner) als Atavismus ansehen, hat nach andern (Driesch, Friedrich, Godelmans, Herbst, Tonsier) je nachdem nur als Hemmungs- oder übermäßige Bildung zu gelten.

Während sich der Widerspruch zwischen Anpassungs- und jener Theorie, welche die Regeneration als Allgemeinerscheinung annimmt, heute bereits zugunsten der letzteren einer Lösung zuneigt, gewinnt man hinsichtlich der "hypotypischen Regeneration " (GIARD) den Eindruck, als sei der Spekulation noch zu viel Spielraum gelassen, weil das Tatsachenmaterial zu gering. Um das bisher Vorhandene durch nene Fakten zu vermehren, wird man zielbewußt nach ihnen suchen müssen; die bisher ermittelten Fälle hypotypischer Regeneration aber sind meist mehr zufällig zutage getreten, indem man die Regenerationsfühigkeit gewisser Organe auf die Probe stellen wollte und unerwarteterweise an ihrer Stelle das nämliche Organ in abweichender Beschaffenheit oder überhaupt andersgeartete Organe erhielt.

Um nun das wünschenswerte planmäßige Suchen nach hypotypischen Regeneraten zu erleichtern, geben die bisher gewonnenen Fälle bereits einige schätzenswerte Winke. Abgesehen von der Rolle, die das Nervensystem dabei spielt, zeigen sie vor allen Dingen, daß es kompliziertere, mehr oder minder einseitig a n bestimmte 
Funktionen angepaßte Organe sind, welche beim Wiederaufbau in andrer, meist vereinfachter Form erscheinen.

Unter ihnen beanspruchen die sekundären Geschlechtsorgane in bezug auf ihr regeneratives Verhalten gesondertes Interesse, weil sie unter jenen Problemen der Entwicklungslehre, die der Bearbeitung seitens der Regenerationsmethodik zugänglich sind, für ein Sonderproblem, das des Sexualdimorphismus, manche Aufklärung hoffen lassen.

Mit einer ziemlich ausgedehnten Arbeit über Regenerationsund Wachstumsgeschwindigkeit der Amphibien beschäftigt, bei welcher der Regenerationsablauf verschiedenster regenerationsfähiger Körperteile quantitativ verfolgt wurde, erzielte ich auch Regenerate solcher Organe, die in dem einen oder andern (zu allermeist männlichen) Geschlecht durch den Besitz sekundärer Sexualcharaktere ausgezeichnet sind. Da die betreffenden neuen Resultate - in qualitativer Betrachtungsweise - mit den erwähnten quantitativen Untersuchungen wenig zu tun haben, also gewissermaßen nur ein Nebenergebnis der letzteren darstellen, berichte ich darüber in einer selbständigen Publikation: um meiner quantitativen Arbeit, deren Veröffentlichung erst bevorsteht, eingereiht zu werden, schienen sie mir doch auf einen zu großen Umfang angewachsen, zumal jene Arbeit ohnehin schon an und für sich einen nicht unbeträchtlichen Raum beanspruchen dürfte. In der hier vorliegenden Sonderpublikation nun, welche sich ausschließlich mit der Regeneration sekundärer Geschlechtscharaktere befaßt, habe ich mich möglichst auf Wiedergabe der bloßen Tatsachen beschränkt, ohne sie eingehender theoretisch zu werten, aber auch ohne mich eines Lapidarstiles zu bedienen, der die Arbeit nur einem Spezialisten des betreffenden Teilgebietes willkommen werden läßt. Im Interesse einer ökonomischen Darstellung hatte ich hier auch keine Veranlassung, den Leser mit langen Versuchsprotokoll en zu ermitiden; in meiner angekundigten größeren Arbeit werde ich ohnehin auf solche zum Zwecke der leichteren Übersicht nicht verzichten können. Ein kurzer Auszug aus dem Versuchsjournal am Ende jedes, die Regeneration eines bestimmten Organes behandelnden Abschnittes dürfte diesmal gentigen, um die nötige Übersicht herzustellen. Von den Ergebnissen wurden nur solche abgebildet, an denen anch auf der bildlichen Darstellung im Vergleich zur Normalgestaltung ein Unterschied deutlich zu erkennen ist.

Den Anstoß aber, gerade sekundäre Geschlechtsmerkmale in 
umfassenderer Weise in jene andern Versuche an Amphibien einbezogen zu haben, verdanke ich den Befunden von BLACKWALL über das bedingungsweise hypotypische Regenerieren der männlichen Palpen bei den Spinnen und denen von Č́ersí ubber das Regenerieren des Begattungsorganes in dem hypotyp gestalteten rechten Tentakel der Sumpfdeckelschnecke.

\section{Darstellung der Versuche.}

\section{A. Froschlurche. Anura.}

1. Die Brunftschwielen an den männlichen Extremitäten.

a) Die Zehenschwielen am Vorderbein von Bufo viridis Laur.: Das Männchen der grünen oder Wechselkröte (Bufo viridis Laur. = variabilis Pall.) trägt zur Paarungszeit - April, Mai am verdickten Daumen einen Ballen, der sowie der Inuenrand des Daumens and des zweiten und dritten Fingers von einer schwarzbraunen Schwiele ibberzogen erscheint, die sich beim Darüberstreichen feilenartig rauh anfuhlt. Außerdem ist die ganze Vorderextremität des Männchens dicker, muskelstärker und mehr einwärts gerichtet, - eine funktionelle Anpassung an den Amplexus bei der Begattung. Da die genannte Krötenart im Garten der Biologisehen Versuchsanstalt massenhaft wild vorkommt und alljährlich in unsre Freilandbassins ablaicht, stellte sie für den beabsichtigten Versuch ein leicht zu erlangendes Objekt vor, das sich auch insofern günstig erwies, als es die nur zur Brunftzeit deutlich werdenden Schwielen in eben jener Zeit mit Bestimmtheit hervorzurufen gestattete, - waren doch die Bedingungen, unter denen diese Tiere zur Fortpflanzung schreiten, reichlich gegeben.

Da bekanntlich die Extremitäten der Froschlurche, abgesehen von wenigen Fällen, nach der Metamorphose nicht mehr regenerationsfähig sind, mußte die Operation behufs Lösung der Frage, ob die Schwielenbildungen, die muskulöse Beschaffenheit und eigentumliche Stellung der Extremität im Regenerat wiedererstehen würden, bei Kaulquappen ausgefuhrt werden, und zwar bei solchen frihen Stadiums, da es sich um die Vorderextremität handelt, welche auch bei nur einigermaßen älteren Larven nicht mehr regeneriert.

Genau den Angaben von Brrwes folgend, auf deren Arbeit ich hinsichtlich der Methode verweise, entfernte ich die noch stummelförmige Anlage der rechten oder beider Vorderextremitäten an Wechselkrötenlarven, die ich aus in Gefangenschaft abgelegten Eiern ge- 
zogen hatte. Natürlich war es damals noch nicht möglich, zwischen Männchen und Weibchen zu unterscheiden, da sich an den Larven keine Merkmale hierftir bieten; doch war vorauszusetzen, daß sich in einer Zahl von 30 operierten Tieren auch Männchen befinden würden, in welcher Voraussetzung ich mich denn auch nicht getäuscht hatte.

Bis zur Metamorphose waren die Regenerate der" Vorderbeine noch deutlich an ibrer geringen Größe zu erkennen; nach der Metamorphose unterschieden sie sich bald nur mehr wenig von normalen. Um aber zu entscheiden, ob auch der sekundäre Geschlechtscharakter wiederum auftreten werde, mußte erst die Geschlechtsreife der jungen Vollkröten abgewartet werden. Zwischen Metamorphose und Geschlechtsreife liegen in der Regel 4 Jahre. Durch erhöhte Temperatur gelingt es, diese Zeit beinahe um die Hälfte einzuschränken, was immerhin seit Erleiden und Ausheilen des Unfalles ein so langes Intervall bedeutet, daß das Resultat eigentlich von vornherein zu erwarten war: in demjenigen Frühjahr, in welchem die jungen Krötenmännchen zum ersten Male ihre trillernde Stimme erschallen ließen, um ein Weibchen anzulocken, bildete sich auch an den ersten drei Zehen ihrer regenerierten Vorderextremität die charakteristische, schwärzlich schwielige Hautwucherung. Der Daumen hatte schon etwas früher durch allmähliche Verdickung und Ballenbildung sein Geschlecht zu erkennen gegeben, ebenso war der Muskelreichtum des Armes und seine nach einwärts gedrehte Stellung nicht zu verkennen.

Auszug aus dem Versuchsprotokoll:

1904. 18. IV. Larven von Bufo viridis dem Ei entschliipft.

13. V. Vorderbeinstummel beiderseits (15 Larven), bzw. nur rechts

(andre Serie von 15 Larven) entfernt.

20.-24. VI. Vorderbeine regeneriert, kleiner als normal.

10.-13. VII. Metamorphose.

1. VIII. Vorderbeine überall fast von Normalgröße.

1906. 3. VIII. Vorderbeine werden besonders muskulos und nach einwïrts gedreht bei 10 Tieren $(\hat{\partial} \vec{b})$ der 1., 9 Tieren der 2. Serie.

1907. 15. II. Verdickung des Daumens in toto wahrnehmbar.

13. III. Bildung des Daumenballens wahrnehmbar.

16. IV. Auftreten der Schwielen.

b) Die Zehen- und Armschwielen von Bombinator pachypus Bp.: Das Männchen der gelbbauchigen oder Bergunke (Bombinator pachypus Bonaparte) stellt im Vergleich zu den übrigen Anuren ein für unsre $Z$ wecke viel angenehmeres Material vor: erstens bleibt das Regenerationsvermögen der Extremitäten bei dieser niedrig- 
stehenden, zur alten Familie der Discoglossiden gehörigen Form uiberhaupt länger erhalten, so daß man keine so jungen Stadien operieren muß; zweitens ist die Schwielenbildung hier nicht auf die Vorderextremität beschränkt, sondern erstreckt sich auch auf die leichter regenerierende Hinterextremität.

Auf dem Vorderbein tritt die schwarzgraue Schwiele nach DürIGEN, S. 547, am Innenrande des zweiten und dritten Fingers, am Innenrande und auf der Oberseite des innersten Fingers oder Daumens, am Daumenballen and an der Innenfläche des Unterarmes auf «, am Hinterbein $\gg$ an der Unterseite der zweiten und dritten Zehe «.

Bezugglich des Wiederauftretens all dieser Schwielenbildungen gilt dasselbe, was von denen der Wechselkröte gesagt wurde, nur mit den Unterschieden, daB ich erstens die Vorderbeine zu einer Zeit amputierte, als ihre einzelnen Abschnitte, einschließlich der Phalangen, unter der bei der Operation zu durchbohrenden Kiemenhöhlendeckhaut schon gut husgebildet waren (Operationsart I bei Bynxes, S. 171), zweitens die Hintergliedmaßen erst knapp vor der Metamorphose abgeschnitten wurden, drittens die Geschlechtsreife schon binnen 11/2 Jahren, vom Ende der Metamorphose an gerechnet, zu erreichen war. Hierdurch war der Zeitraum zwischen Verlust des Körperteiles und Auftreten seiner Sexualcharaktere erheblich verringert, ohne jedoch dem letzteren auch nur den mindesten Eintrag zu tun. Sowohl am regenerierten Hinter- als auch am Vorderbein kamen sämtliche Hautwucherungen zum Vorschein, hier nicht nur die Zehenschwielen, sondern anch die Armschwiele.

Auszug aus dem Versuchsprotokoll:

Zwei Serien mit Hinterbeinamputationen.

1905. 2. VI. Larven aus dem Ei entschliipft.

15. VIII. Hinterbeine beiderseits (15 Larven), bzw. nur rechts (andre Serie von 15 Larven) amputiert.

12.-17. IX. Hinterbeine regeneriert, kleiner als normal.

14.-18. IX. Metamorphose.

25. IX. Hinterbeine durchweg von Normalgröße.

1907. 6. V. Auftreten der Schwielen bei 8 Exemplaren ( $\delta$ ) der 1., 5 der 2. Serie.

Zwei Serien mit Vorderbeinamputationen.

1905. 15. VIII. Vorderbein beiderseits (1ó Larven', bzw. nur links (andre Serie von 15 Larven) amputiert.

14.--17. IX. Vorderbeine regeneriert, kleiner als normal.

15.--19. IX. Metamorphose.

1. $\mathrm{X}$. Vorderbeine fast von Normalgröße.

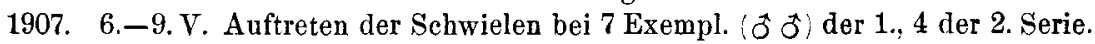




\section{Die Schallblasen der Männchen.}

a) Der Kehlsack von Hyla arborea L.: Bekanntermaßen sind die Geschlechter des europäischen Laubfrosches (Hyla arborea Linné) jederzeit daran za unterscheiden, daß das Männchen eine von der ubrigen, weißen Unterseite abstechende dunkle, gelblich- bis graubraune, meist schmutzig lederfarbene Kehlhaut besitzt, die im Ruhezustand in viele Längs- und Querfalten zusammengezogen erscheint, beim Quaken aber zu einer kugligen Schallblase aufgebläht wird. Die Kehle des Weibchens hingegen ist einfach weiß, gleich der übrigen Ventralseite, höchstens grau angeflogen.

Ich operierte zunächst $\mathbf{j u n g v e r w a n d e l t e ~ L a u b f r o ̈ s c h e , ~ u n d ~}$ zwar notgedrungen Männchen und Weibchen ohne Unterschied, da an deren Kehlhaut das erwähnte Merkmal fehlt: sie haben noch 2 bis 3 Jahre bis zur Geschlechtsreife zu warten. Mit einer gekrümmten, scharfen, vor dem Gebrauch natürlich jedesmal in der Flamme zu sterilisierenden Schere schnitt ich die Kehlhaut längs des Unterkieferrandes weg, wobei man sehr darauf achten muß, die Zunge nicht zu verletzen, schlug die abpräparierte Haut dann zurïck und entfernte sie durch einen Transversalschnitt an der Grenzlinie, bis wohin etwa beim geschlechtsreifen Tiere die braune Färbung gereicht hätte.

Zwei Monate and etwas dartiber nach dieser immerhin schweren Operation war eine zarte neue Haut über die Kehle gespannt, welche natürlich nun die weiße Farbe aller jungen Laubfroschkehlen aufwies. Nach $13 / 4$ Jahren aber, innerhalb welcher Zeit in Anbetracht der hohen Gefangenschaftstemperatur (16 bis 18 Grad C.) die Geschlechtsreife erreicht schien, färbten sich die Kehlen aller Männchen unter ihnen, bei operiert gewesenen Exemplaren nur etwas später als bei unverletzt gebliebenen Kontrolltieren, zuerst gelblich, dann bräunlich. Die Laubfroschmännchen mit regeneriertem Kehlsack gebrauchen diesen heute ganz ebenso zur Stimmverstärkung, als hätten sie nie einen Defekt daran erlitten. Es ist zu betonen, daß in diesem Versuch der Zeitraum zwischen Operation und Auftreten der erwarteten Merkmale am Regenerat abermals im Vergleich zum vorigen Versuch ein kürzerer war; trotzdem reichte er noch aus, um von vornherein keine Hypotypie erkennen zu lassen, sondern gleich nur hier bereits ein wenig verspätet — vollständig zu liefern, was die Männchen als solche fordern konnten. Um zu sehen, ob nicht doch etwa ein Teil derjenigen Laubfrösche, die endgültig nur eine 
weiße, glatte Kehle bekommen hatten, Männchen seien, sezierte ich sie und fand sie durchwegs weiblichen Geschlechts. Ein Verfahren, welches ich übrigens schon in den vorigen Versuchen bei den Kröten und Unken, die ohne Schwielen geblieben waren, mit gleichem Ergebnis angewendet hatte.

Werber bat bei $2 \frac{1}{2} \mathrm{~cm}$ langen, demnach etwa ein Jahr alten Laubfröschen noch Kieferregenerate erhalten, was bei doppelt so großen schon nicht mehr gelang. Da bei meiner Operation nicht der Wiederersatz eines Knochens beansprucht wurde, wollte ich aber doch auch einen Versuch wagen, sie an Laubfröschen zur Ausführung zu bringen, deren Kehlsack bereits fertig differenziert war. Ich ging dabei einerseits in der zuvor angegebenen Weise, also mit $\mathrm{Ab}$ lösung der ganzen Kehlhaut, soweit sie braun ist, zu Werke; anderseits machte ich noch eine leichtere Operation, indem ich die faltige Hant mit Zeigefinger und Daumen faßte und bis zu der Ausdehnung vorzog, welche sie beim Quaken einnimmt, und dann mit einem einzigen Schnitt einer größeren, langschneidigen Schere den unteren Teil des Sackes entfernte. Auf diese Weise war nur der mittlere Teil der Kehlhaut in Form eines kreisförmigen Ausschnittes entfernt, während an den Kieferrändern und an der Grenze gegen die Brust zu ein Streifen brauner Haut zurückblieb. Ich hatte diese zweifache Operation hauptsächlich ans dem Grunde unternommen, weil an die Möglichkeit zu denken war, daß im ersten Falle (bei Totalablösung) ein hypotypisches, im letzteren Falle (bei Partialablösung) gleich ein vollkommenes Regenerat zustande kommen würde. Außerdem konnte die stärkere Operation bei schon geschlechtsreifen Tieren vielleicht überhaupt nicht mehr gelingen, die schwächere aber wohl.

Indessen es führten beide Operationen zum Ziele, und zwar zu gleichem Endziele. Nur der Verlauf war ein etwas verschiedener. Die total abgelöste Kehlhaut regenerierte binnen durchschnittlich 50 Tagen, die nur eines Mittelstückes beraubte binnen 35 bis 40 Tagen so weit, daß die Verletzung wiederum vollständig ausgeebnet erschien. An Stelle der braunen, faltigen Haut war aber in beiden Fällen eine weiße und glatte (d. h. auf vorgeschrittenem Stadium der Wundheilung wohl granulierte, aber nicht gefaltete) Haut gewachsen. Die total operierten männlichen Laubfrösche waren nun äußerlich mit Weibchen zum Verwechseln ähnlich, die partiell operierten hatten zunächst einen runden, weißen Fleck in brauner Umsäumung.

Allein durchschnittlich 5 Wochen waren seit der Operation ver- 
flossen, als sich jene braune Umsäumung des weißen Mittelfeldes zurückbildete: sie verblaßte, die Falten verschwanden. Schließlich hatte sie gelblichweißes Aussehen gewonnen, die ganze Kehlhaut war somit abermals der eines Weibchens ähnlich.

Etwa 1 Monat lang behielt die Kehlhant in beiden Versuchsreihen dieselbe Beschaffenheit, dann begann von der Mitte aus eine dunklere Pigmentierung aufzutreten, zuerst in Form eines schwer zu bezeichnenden, grïnlichgelben Anfluges, der sich allmäblich uber die Kehle ausbreitete und zugleich im Centrum immer mehr verdunkelte. Dieses war schon dunkel olivenbraun, die Randpartien noch hell lederfarben, braungelb. Auch die Falten traten wieder hervor, indem das Wachstum der Kehlbaut anscheinend von dem Momente an, als sie imstande war, den Raum zwischen beiden Unterkieferästen zu bespannen, keineswegs aufgehört hatte, sondern zuerst bis zur Bildung von Quer-, dann auch von Längsfalten seinen Fortgang nahm. 3 bis 4 Monate nach der Operation hatten alle Laubfroschmännchen ihr ursprüngliches Aussehen und ihre Stimmfähigkeit wiedererlangt.

Auch letztere hatte einen RegenerationsprozeB durchgemacht. Die Tiere waren nämlich von dem Augenblicke an, als die Wunde verheilt war, nicht etwa stumm geblieben, sondern hatten bereits wieder zu quaken begonnen. Da aber keine Schallblase vorhanden war, obzwar die Frösche alle Anstrengungen machten, die noch straffe Kehlhaut möglichst aufzublähen, klangen die Stimmen anders als sonst, viel schwächer, ähnlich einem Ziegenmeckern. Gewiß mag diese rege funktionelle Inanspruchnahme der regenerierenden Schallblase ihre rasche und vollständige. Wiederherstellung wesentlich befördert haben.

Ein Wort möchte ich noch bezüglich der Fütterung der operierten Laubfrösche nachtragen. Nur bei denen mit total abpräparierter Kehlhaut waren besondere Maßnahmen nötig, die andern fraßen die ganze Zeit über freiwillig. Jene aber konnten, ehe nicht ein vorgeschrittenes Heilungsstadium erreicht war, nicht selbst fressen. Obzwar die Hungerperiode, wenn ich innerhalb jener Zeit gar keine Vorsorge für die Nahrungsaufnahme getroffen hätte, auch keine so lange gewesen wäre, um die Tiere ernstlich zu gefährden, machte ich doch im Interesse eines möglichst günstigen, normalen Heilungsablaufes von einem System der künstlichen Fitterung Gebrauch, daß ich schon vor mehreren Jahren (1900) genau beschrieben, und dessen sich auch WERBER bei seinen Kieferamputationen bedient hat: durch vorsichtiges, maßvolles Stopfen der Tiere mit dekapitierten Mehlkäfer- 
larven ist es möglich, sie ununterbrochen im Vollbesitze ihrer Kraft und Wachstumsintensität zu erhalten.

\section{Auszug aus dem Versuchsprotokoll:}

Serie von 20 frisch verwandelten Laubröschen.

1904. 29. VI. Metamorphose.

30. VI. Abtragen der Kehlhaut.

1.-8. IX. Nene Kehhaut gebildet.

1906. 20.-24. V. Verfäbung der weißen Kehle in Braun bei 11 operierten,

9.-14. V. $\quad$ - $\quad-\quad$ - $\quad$ - $\quad$ - $\quad$ - $\quad$ - nicht operierten Laubfrüschen $(\hat{\partial} \hat{\partial})$.

Totalablüsung der Kehlhat geschlechtsreifer Laubfrosch-öす.

1904. 30. VI. Kehlbaut von 10 Exemplaren ganz abpräpariert.

18.-21. VIII. Regeneration des Kehlsackes, weiß und glatt.

20.-23. IX. Beginn der Faltung und Verfärbung des Kehlsackes in Braun.

Partialabtrennung der Kehlhaut geschlechtsreifer Laubfrosch- $\widehat{\jmath}$. 1904. 30. VI. Ein rundes Stiick aus der Kehlhaut geschnitten bei 10 Exempl.

3.-8. VII. Das ausesechnitten gewesene Mittelfeld von weißer, gespanter, faltenloser Haut überkleidet.

7.-9. VII. Beginnende Ausbleichung und Glätung der umgebenden, bei der Operation stehengebliebenen, braun gewesenen Haut.

8.-10. VIII. Begrinnende Wiederverdnnkelung nud Faltung des Kehlsackes vom Centrum her.

b) Die doppelte Schallblase von Rana esculenta L.: Das Männchen des Teichfrosches (Rana esculenta Linné) besitzt »zwei sehr entwickelte Stimmsäcke, welche im luftgefüllten Zustande als zwei milchweiße oder graue, erbsen- bis kirschengroße kuglige Blasen hinterm Mundwinkel und unterm Trommelfell hervortreten, während im luftleeren Zustande der Schallblasen die änßere, verdünnte Körperhaut an Stelle der früheren Hervortreibung eine Art Tasche nach einwärts bildet, deren Eingang als ein mit dem Unterkiefer gleichlaufender Längsschlitz sich zeigt (Dürigen, S. 424).

Nimmt man einen männlichen Teichfrosch in die Hand und übt in dorso-ventraler Richtung einen sanften Druck aus, so gelingt es leicht, die Schallblasen zum Vorschein zu bringen: der Frosch läßt meckernde Laute hören und stülpt die beiden vibrierenden Säcke an den Mundwinkeln nach außen. Auch mit einer Pinzette lassen sie sich unschwer herworziehen. In einem Behälter unsrer Anstalt leben seit langem 6 männliche Teichfrösche, die einer italienischen Abart (var. lessonae Boulenger) angehören und bei denen es besonders leicht ist, die Schallblasen hervorzudrängen: schon beim bloßen Anfassen ist dies regelmäßig der Fall. Außerdem waren diese Frösche im 
Vergleich zu der in Gefangenschaft, abgesehen von einem dumpfen Knurren, meist ziemlich schweigsamen Rana esculenta typica Boul. sehr sangesfreudig, so daB sie für die beabsichtigte Operation in jeder Beziehung am geeignetsten zu sein schienen. Da ihre Zahl für einen Regenerationsversuch im allgemeinen als zu gering anzusehen ist, wurden aber außer ihnen noch etliche Männchen der forma typica herangezogen. Die Operation erfolgt einfach dadurch, daB man die durch Druck oder mit Hilfe der Pinzette ausgestülpten Stimmsäcke möglichst an ihrer Ursprungsstelle mit einem einzigen Scherenschlag abzwickt. Die schwach blutenden Reste werden eingezogen und der Frosch sieht aus wie zuvor.

Um den Verlauf des Regenerationsprozesses zu verfolgen, versuchte ich nach 2,3,4 Wochen dasjenige, was von den Schallblasen bereits wieder vorhanden sein mochte, mit der Pinzette nach außen zu befördern; die Druckmanipulation war nämlich jetzt erfolglos. Nach der zweiwöchentlichen Ruhepause war gar nichts zum Vorschein zu bringen und die eingeführte Glassonde drang bis in die Mundhöhle durch, ein Beweis, daß sich noch kein geschlossener Sack neu gebildet hatte. Nach 3 Wochen ermittelte die Sonde das Vorhandensein eines blindgeschlossenen Sackes, der sich aber nicht nach außen ziehen ließ; Gewalt wollte ich nicht anwenden, um ein abermaliges Zerreißen der, wie ich meinte, im jetzigen Zustand gewiß besonders zarten Gebilde zu vermeiden. Nach 4 Wochen und etlichen Tagen gelang es, je einen Sack, der das Regenerat der Schallblase darstellte, vorzustülpen, welcher Sack im Vergleich zu den alten Schallblasen kleiner war und nicht aus jener zarten, durchsichtigen, sondern im Gegenteil aus einer derberen, kanm durchscheinenden Haut bestand. Die Frösche machten aber auch gar keinen Versuch, ihn mit Luft aufzublähen, daher mochte die mit der starken Dehnung Hand in Hand gehende Verdünnung ausgeblieben sein. Darin liegt ein Gegensatz im Verhalten des Laub- und Wasserfrosches: auch die Italiener unter den Teichfröschen habe ich, obzwar ihre Stimmsäcke verheilt waren, seit der Operation nicht mehr schreien gehört.

Auszug aus dem Versuchsprotokoll:

1905. 1. VI. 20 Teichfroseh- $\partial \quad 6$ davon var. lessonae) die Stimmsäcke abgeschnitten.

21. VI. Fertigstellung des Verschlusses bei der Revision konstatiert.

3. VII. Regeneration kleiner, grober Hautsäcke an Stelle der Schallblasen. 


\section{B. Schwanzlurche. Urodela.}

1. Die Kämme der männlichen Wassermolche.

a) Ganzrandige Kämme: $\alpha$ ) Triton alpestris Laur. - Der männliche Bergmolch (Triton [Molge] alpestris Laurenti) trägt inter nuptias einen im Nacken beginnenden, die Ruickenmedianlinie entlangziehenden, ohne Unterbrechung auf die Schwanzkante übergreifenden, ganzrandigen Hautsaum von hellgelber Grundfarbe mit schmalen, senkrechten, schwarzen Querbinden (Taf. II Fig. 3). Will man die Regenerationsfähigkeit dieses Hochzeitsattributes untersuchen, so muß man die Operation zu Beginn der Laichzeit (bei Wien im Februar und März) ausführen. Denn nur so darf man, vorausgesetzt daß die Molche unter giinstigen Lebensbedingungen gehalten werden, darauf rechnen, daß die abgeschnittenen Kämme sich im Verlaufe derselben Laichperiode noch einmal erheben. Im andern Falle, wenn die Operation schon mit dem Einsetzen des natürlichen Schrumpfungsprozesses zusammenfällt, kann man zwar mit Hilfe künstlicher Mittel, von denen gleich die Rede sein wird, immer noch Resultate bekommen, falls die Molehe im Wasser bleiben; kriechen sie aber ans Land, was sie nach der Fortpflanzungszeit gern tun, ohne daß man es, sollen die Tiere nicht zugrunde gehen, verhindern kann, so ist auf eine abermalige Bildung des Kammes erst im nächstfolgenden Frühjahr mit dem Neueintreten der Paarungslust zu hoffen.

Die nächstliegende Operation besteht darin, mit Hilfe einer kleinen Schere, deren schneidende Hebelarme gerade und recht kurz sein sollen, den Kamm.vom Nacken angefangen bis zur Schwanzspitze in continuo möglichst nahe am Rumpf abzuschneiden, wobei man den Körper des Molches seiner ganzen Länge nach zwischen dem gestreckten Zeigefinger, dritten Finger und Daumen in gerader Stellung festhält. Variationen des Versuches ergeben sich daraus, daß man entweder nur den Rücken- oder nur den Schwanzkamm, diesen außer auf der Dorsal- auch auf der Ventralkante oder nur auf einer von beiden allein wegschneidet, sowie, daß man den Schwanzsaum zugleich mit dem Muskel- und Skeletteil des Schwanzes zur Hälfte oder zu zwei Drittel amputiert. Diese Variationen wurden bei allen beztiglich ihres Kammes zur Untersuchung gelangten Tritonen durchgefuhrt.

Ich versuchte, den Einflu $B$ des Geschlechtstriebes auf die Regenerationsgeschwindigkeit des Kammes festzustellen, indem ich jenen auf verschiedene Weise zu steigern unternahm. Zuerst dadurch, daß ich nichtoperierte, brünftige Weibchen mit den 
männlichen, operierten MoIchen im selben Aquarium hielt, die uibrigens, wie viele operierte Tiere, an und für sich schon einen besonders heftigen Geschlechtstrieb an den Tag legten. Wie ich jedoch wohl im vorhinein hätte wissen können, war das Zusammenbringen der Männchen und Weibchen nicht der richtige Weg, Kammregenerate zu erlangen. Der Geschlechtstrieb wurde dadurch zunächst zwar erhöht, aber auch um so rascher befriedigt und beendigt: innerhalb einer Zeit, die kaum zum Wundversehluß hinreichte, setzten die Männchen, jedes einzelne zu wiederholten Malen, ibre Spermatophoren ab, worauf sie indifferent warden und ihre Hochzeitskleider verblaßten.

Es handelte sich nämlich, un zum Ziele zu kommen, nicht darum, den Fortpflanzungstrieb zu erhöhen, um ihn alsbald zu befriedigen, sondern ihn im Gegenteil recht lange unbefriedigt auf seiner vollen Höhe zn erhalten. Ich setzte deshalb die Weibchen zunächst in besondre Glaswannen und stellte diese dicht neben jene, in denen die Männchen untergebracht waren. Diesmal ging es besser: die Männchen des Versuchsbeckens waren durch die Weibchen, welche sie durch die Glaswände erblickten, erregt, sammelten sich an der Glaswand, welche ans Nachbarbecken anstieB, und gaben durch das charakteristische Umbiegen und Wedeln des Schweifes ihre Begattungslust zu erkennen. Trotzdem die Vereinigung nicht möglich war, setzten aber die Männchen, gegenüber der vorgenannten Versuchsaufstellung nur wenig verzögert, ihre Spermatophoren ab, bevor neue Kämme gewachsen waren, weshalb sich auch dieses Mittel, obgleich zweckentsprechender als die unmittelbare Vereinigung mit dem andern Geschlecht, auf die Dauer doch nicht recht brauchbar war.

Ein andres Mittel, die Hochzeitsattribute der Tritonen stärker zur Geltung zu bringen, besteht darin, sie in möglichst luftreichem Wasser zu halten. De Bedriaga beobachtete, daß der in pyrenäischen Hochgebirgsseen lebende Triton [Euproctus] asper Dugès sofort Brunfterscheinungen aufwies, wenn er in sein Wohnbecken ei skaltes Wasser einfließen ließ (S. 736, 738), eine Erscheinung, die wenigstens teilweise neben dem Temperaturfaktor dem größeren Luftreichtum kalten Wassers zuzuschreiben sein dürfte. KoHLwey (S. 32) brachte eine Gesellschaft von Tritonen im Winter in reinen Sauerstoff: »In wenigen Minuten wurden all die gelben, roten und violetten Flecke sichtbar, welche sonst nur im Glanz der Früblingssonne hervorgerufen werden*. Da ich reinen Sauerstoff momentan nicht zur Verfügung hatte, versuchte ich es erst mit dem vorgenannten Mittel: ich setzte die Molche in durchfließendes Hochquellen- 
wasser von $6-7^{\circ} \mathrm{C}$. Eine andre Partie an ihren Kämmen operierter Molche setzte ich in ein Aquarium, in welches ein Ausströmungskörper der Luftleitung mündete, so daßs dort das Wasser zwar nicht abgekiuhlt, wohl aber bei $18-20^{\circ}$ beständig von zahllosen feinen Luftblasen durchströmt und hierdurch reichlich mit atmosphärischer Luft geschwängert wurde. Beide Maßregeln sind offenbar von Nutzen begleitet gewesen, denn ich fand Ende Mai, zu einer Zeit, als die normal gehaltenen, nicht operierten Triton alpestris ihr Hochzeitskleid schon mit der einfacheren Tracht post nuptias vertauscht hatten, regenerierte Kämme vor, und ebenso standen auch die von der Operation nicht betroffenen Attribute der Paarungszeit, die Farbe und der Hautsaum an der Oberlippe, noch fast auf dem Höhepunkte ihrer Entfaltung. Da dies jedoch auch bei zwar normal gehaltenen (nicht in Durchluftung oder fließendes Wasser gesetzten), aber operierten Tieren zum Teil der Fall war, so ist viel Wahrscheinlichkeit vorbanden, daß schon die Operation selbst, wie noch unpublizierte Beobachtungen andrer Mitarbeiter unsrer Anstalt mir bestätigen werden, einen Reiz in der entsprechenden Richtung hin ausübt (vgl. Kammener, 1907 , S. j17 unten).

Nicht beim Bergmolch zwar, sondern bei einer südlichen, später laichenden Molchspecies (Triton marmoratus) versuchte ich schließlich noch im Juli die Wirkung des reinen Sauerstoffs, den ich, durch einen Ausströmungskörper aus Kunststeinmasse zerstäubt, in Form eines zarten Bläschenschleiers in das Aquarium eintreten ließ. Der Versuch gelang über Erwarten: ich erhielt Regenerate des Kammes, wo ich für heuer die Hoffnung bereits aufgegeben hatte. Ob es auch bei den andern, frühlaichenden Molchen -2 bis 3 Monate nach ihrer Laichperiode - noch gegangen wäre, weiß ich nicht; ich hatte für die jetzt zu unternebmende Arbeit, da schon im Besitz von hinlänglich vielen Regeneraten, keine Veranlassung mehr, es zu versuchen.

Um nun zur Darstellung des Versuchsablaufs beim Bergmolch zurückzukehren, ist zunächst festzustellen, daß sich als Anfang des Regenerationsprozesses 5 bis 6 Tage nach der Operation ein zarter, durchscheinender, weißlicher Hautsaum anf dem Ruickenfirste erhob, der vorerst die Querbänderung nicht aufwies und, wie der primäre Kamm, ganzrandig war. In der zweiten Woche begannen die schmalen, schwarzen Querstreifen vom Rumpf aus, wo punktförmige Reste derselben stehen geblieben waren, auf den neugebildeten, bis dahin einfarbigen Kamm uberzuwachsen. Anfangs waren sie blaß, 
graubraun, und erst allmählich nahmen sie die tintenschwarze Farbe wie auf dem normalen Kamm an. Die neuen Querbinden verliefen nunmehr nicht durehwegs in der streng dorso-ventralen Richtung wie ursprünglich, sondern waren an mehreren Übergangsstellen von den bei der Operation stehengebliebenen Resten zu den neugebildeten Streifen abgebogen oder sogar abgeknickt, - eine Unreg elmäßigkeit, die sich auch allmählich ausgleicht. Ein Regenerationsstadium, auf welchem die neuen Querbinden noch nicht so intensiv gefärbt sind wie beim Normaltier, stellt Figur $3 a$ dar. Vom Abschneiden des Kammes bis zu seiner in Form und Farbe vollkommenen Wiederherstellung waren knapp 3 Wochen vergangen. Die vorhin aufgezählten Variationen der Kammamputationen (Abschneiden bestimmter Partien) ändern an dem Ergebnis nichts wesentliches; sie rufen nur Verschiebungen in der Dauer des Prozesses hervor, von denen diejenige bei Amputation ganzer Schwanzteile naturlich am größten ist. Die Verbleichung der Färbung und Zeichnung greift, wo nur gewisse Kammpartien zu regenerieren waren, vorïbergehend auch auf die unverletzt gebliebenen Nachbarpartien streckenweise über.

Einer Erscheinung ist aber noch Erwähnung zu tun, die sich dann auch beim Regenerieren der Kämme andrer Tritonarten einstellte und die besonderes Interesse verdient: der neue Kamm ist nämlich mit seinen $3 \mathrm{~mm}$ entschieden höher als der primäre Kamm, welcher beim Bergmolch selten über $2 \mathrm{~mm}$ erreicht. Es ist also ein neuer Fall von hypertrophischer Regeneration zu konstatieren, die bisher nur an wenigen Beispielen - Oligochaeten (KonschelT), Wasserassel (Zuelzer), Fischflossen (Buschisiex) - bekannt war.

Auszug aus dem Versuchsprotokoll:

1907. 1. III. Abschneiden des $2 \mathrm{~mm}$ hohen Triton alpestris-Kammes seiner ganzen Lünge nach, nur auf dem Rïcken, nu: auf dem Schwanze und Amputation der distalen Schwanzhälfte bei je $10 \hat{f} \vec{s}$.

7., ๖., 5., 17. III. Regeneration eines farblosen Saumes im 1., 2., 3., 4. Fall (hier auf dem Regenerat der distalen Schwanzhälfte.

11., 8., 7., 20. III. Nenbildung der Querstreifen im 1., 2., 3., 4. Fall.

21., 20., 21. III., 14. VI. Regenerate vollendet gleich aussehend wie die primären Teile im 1., 2., 3., 4. Fall.

30. III. Der neue Kamm höher als der frihere nur bei Entfernung des ganzen Kammes beobachtet, $3 \mathrm{~mm}$.

p) Triton marmoratus Latr. - Auch der männliche Marmor molch (Triton [Molge] marmoratus Latreille) trägt einen ganzrandigen, über der Cloake seicht eingesattelten Kamm, der schon zwischen den Augen beginnt und bis zu der ansehnlichen Höhe von $7 \mathrm{~mm}$ ansteigt 
(Fig. 1). Seine Grundfarbe ist hell- bis orangegelb, seine Zeichnung besteht, ähnlich wie bei Triton alpestris, aus senkrechten, schwarzbraunen Querstreifen, die aber auf dem Schwanzkamm kompliziertere, verschnörkelte Figuren annehmen.

Ich erhielt das Material, an welchem ich die Versuche anstellte, verhältnismäßig spät im Jahre (20. V. 07), und die eingefallenen Bäuche der Weibchen bewiesen mir, daß das Laichgeschäft schon vorïber war. Immerhin trugen mehrere Männchen noch ihre Kämme, die während des Transportes, der außer Wasser, in nassen Pflanzen, stattgefunden hatte, zwar am Rande etwas verschrumpft waren, sich aber im Wasser bald wieder zu einer für die Operation brauchbaren Höhe empor richteten.

Um sie durch den natürlichen Geschlechtstrieb nach der Operation wieder wachsen zu lassen, war es aber doch zu spät. Die Operation hatte am 20. V. stattgefunden, und am 15. VII. traf ich die Tiere immer noch ohne jeden Kamm an; auch anf den nicht operierten Partien war er versehwunden; nur die Wundränder hatten sich über der beim Abschneiden zurückgelassenen Leiste geschlossen. So versuchte ich es denn, das Wasser mit reinem Sauerstoff zu durchschwängern, und zwar, wie schon im Abschnitte über Triton alpestris vorausgeschickt, mit giinstigem Erfolg. Denn im Zeitraum weniger Tage regenerierten nunmehr Kämme, ungefähr gleich den alten: sie waren ebenfalls ganzrandig, in ihrer Farbe noch blaß, nämlich weißlichgelb mit hellbraunen Streifen, bzw. am Schwanze mit hellbraunen Schnörkeln, die im Gegensatz za Triton alpestris gleich am Beginn der Regeneration auftraten, und 4 bis $5 \mathrm{~mm}$ hoch waren. Auch wo der Kamm nicht seinem ganzen Verlaufe nach abgeschnitten worden war, erhob er gich auf den nicht operierten Körperpartien von neuem, hier aber sofort in der ursprïnglichen Farbensattheit. Beim Niederschreiben dieser Zeilen (7. VIII.) ist die alte Farbenintensität und Höhe der regenerierten Kammpartien zwar noch nicht erreicht, aber auch ihr Wachstum noch nicht abgeschlossen (Fig. 1a). Die Sauerstoffzufuhr war am 30. VII. eingestellt worden.

Schon bei früherer Gelegenheit (1905) habe ich darauf aufmerksam gemacht, daß Triton marmoratus im Gegensatz zu den Angaben Schreibers, Fraisses und Weismanns durchaus nicht schlechter regeneriert als beispielsweise sein durch hohes Regenerationsvermögen geradezu berühmter Verwandter, der Triton cristatus. Was ich damals für Schwanz und Beine nachweisen konnte, ist nun auch für den Kamm dargetan, von welchem ScHrEiber selbst kleine De- 
fekte nicht mehr repariert werden sah (wahrscheinlich eben deshalb, weil es so kleine Defekte waren, während größere za einer viel bedeutenderen Wachstumbeschleunigung Veranlassung geben). Es kann nicht oft genug betont werden, daß solch scheinbare Regenerationsunfähigkeit bei Tieren, deren nächste Verwandte bekannt gute Regeneratoren sind, stets nur auf sekundäre Ursachen, ungunstige Lebens-, namentlich Temperaturbedingungen, und in den allermeisten Fällen auf leichte Infizierbarkeit der Wunden zurückzuführen ist. Die große Schnelligkeit aber, mit der die abgeschnittenen Kämme wieder emporschießen, wenn nur der zu ihrer auch sonst raschen Entstehung unentbehrliche Anreiz, der Geschlechtstrieb rege ist, gewährt eine neuerliche glänzende Bestätigung für PrziвкAмs Wachstumstheorie der Regeneration. Die Kämme in ihrer Eigenschaft als einfache, schnellwüchsige Hautduplicaturen können bei der Regeneration eine Beschleunigung ihres Wachstums natürlich eher leisten als Gliedmaßen oder andre Körperteile mit Einschlïssen starrer, höher differenzierter Gewebsarten.

Auszug aus dem Versuchsprotokoll:

1907. 20. V. Abschneiden des $7 \mathrm{~mm}$ hohen Triton marmoratus-Kammes seiner ganzen Länge nach, nur auf dem Rücken, nur anf dem Schwanze, Amputation der distalen Schwanzhälfte bei je $3 \precsim 3$.

15. VII. Distale Schwanzhälfte regeneriert (4. Fall), aber nirgends Kämme, da Laichperiode voriber.

18. VII. Beginn der Sauerstoffdurchlïftung.

24., 23., 22., 23. VII. Regeneration des Kammes im 1., 2., 3., 4. Fall, Regenerate $4-5 \mathrm{~mm}$ hoch, von Anbeginn gefärbt, nur blasser als normal:

30. VII. Einstellung der Sauerstoffzufuhr.

7. VIII. Kämme ỉberall $5-6 \mathrm{~mm}$ hoch, noch immer blasser als normal.

1) Triton blasii De l'Isle. - Der Blasius sche Kammolch (Triton [Molge] blasii De l'Isle) ist, nachdem man schon lange seine Bastardnatur vermutet hatte, endlich von WoLTersTorfF experimentell als Bastard von Triton marmoratus und Triton cristatus entlarvt worden. Schon aus diesem Grunde mußte diese interessante Molchform ein besonders willkommenes Objekt auch für Regenerationsversuche darstellen, und ich bin daher meinem verehrten Freunde Dr. W. WolterstorfF besonders dankbar, eine ganze Reihe von ihm aus Triton marmoratus-Männchen (Fundort Argenton) und Tr. cristatus subsp. carnifex-Weibchen (Neapel) gezüchteter Exemplare meinen Untersuchungen zur Verfügung gestellt zu haben.

Schon im Februar waren die Bastardmännchen im Vollbesitze 
ihrer Brunftmerkmale und behielten dieselben bis in den Mai. Einigemal wurden auch Eier abgelegt, die aber verpilzten und - vielleicht nur deshalb - nicht zur Entwicklung gelangten. Die Kämme waren denen von Triton marmoratus in bezug auf ihre Gestalt ungemein ähnlich, unterschieden sich aber von ihnen dadurch, daß sie uber der Schwanzwurzel eine tiefere, sattelförmige (doch nicht wie bei $T r$. cristatus bis auf den Rumpf reichende) Einsenkung zeigten. In ihrem Verlauf waren die Kämme, die an ihrer höchsten Stelle $8 \mathrm{~mm}$ erreichten, merklich bogenförmig ausgeschweift, aber durchaus nicht gezackt (Fig. 2).

Nun ist daran zu erinnern, daß die väterliche Stammform, Triton marmoratus, wie schon beschrieben, einen ganzrandigen, die mütterliche Stammform, Triton cristatus (natürlich nur im männlichen Geschlechte) einen tief ausgezackten, gesägten Kamm besitzt; der sanft ausgeschweifte primäre Kamm des männlichen Bastards kann aber noch als ganzrandig bezeichnet werden.

Ganz anders der regenerierte Kamm des Bastards: er zeigt weder einen ganzrandigen, noch einen ausgeschweiften, noch einen zackig gesägten Rand, sondern ist - um abermals einen der hier sehr gut passenden, vom Blattrand gebrauchten botanischen Ausdricke anzuzuwenden - feinkerbig (Fig. 2a). Während also der primäre Kamm von Triton blasii sich mehr demjenigen von Triton marmoratus nähert, ist der regenerierte Kamm demjenigen des Triton cristatus ähnlicher. Das Regenerat ist demnach hier kein hypo-, sondern ein hypertypisches: es ist differenzierter als die primäre Bildung. Für diese Tatsache kann wohl kaum etw̧as andres als die Ahnenschaft des Triton cristatus zur Erklärung herangezogen werden; daß Triton cristatus im weiblichen Geschlecht, als Mutter, an der Abstammung meiner Triton blasii beteiligt ist, kann jener Auffassung selbstverständlich nicht hinderlich sein. Es liegt somit ein Wechsel in den Elternmerkmalen, bedingt durch Regeneration, vor, welcher dadurch beweiskräftiger wird als die bisher dafür angesprochenen Fälle, daß bei ihm die Stammformen durch sorgsam beaufsichtigte Zuichtung genan bekannt sind.

De Bedriaga gibt bezüglich des Kammes von Triton blasii an, er sei »unregelmäßig und fein ausgezackt* (S. 672), was für meine Exemplare ursprünglich nicht zutraf, wohl aber nachdem sie die Kämme regenerativ nochmals erzeugt hatten. Bei der großen Variationsbreite aller Bastardformen könnte eine solche Verschiedenheit nicht Wunder nehmen. Immerhin möchte ich noch eine 
andre Möglichkeit des Zustandekommens *unregelmäßig* gezackter Kämme bei den De Bedriaga vorgelegenen Exemplaren ins Auge fassen: es könnte sich um streckenweise regenerierte Kämme gehandelt haben; kommt es doch unzähligemal vor, daß die Tritonen sich aus Futterneid oder Eifersucht an jenen Hautwucherungen packen und verwunden.

Durch die bei Besprechung von Triton alpestris erwähnten Versuchsvariationen habe ich mich dann noch überzeugt, daß der Kamm streng nur dort das gezackte Aussehen gewinnt, wo er verletzt wurde; die nicht verletzten Partien bleiben ganzrandig, ja selbst die ausgeschweiften Linien an ihnen strecken sich zu schnurgeraden Linien.

Auszug aus dem Versuchsprotokoll:

1907. 28. II. Abschneiden des $8 \mathrm{~mm}$ hohen, seicht ausgeschweiften Triton blasiiKammes seiner ganzen Länge nach, nur auf dem Rücken, nur anf dem Schwanze und Amputation der distalen Schwanzhälfte, bei je 1 o.

כ., 4., 4., 20. III. Regeneration eines von Anbeginn gefïrbten und gezeichneten, fein gekerbten Saumes im 1., 2., 3., 4. Fall (hier auf dem Schwanzregenerat;.

10. III. Regenerat des 1. Falles 7, des 2. Falles 6, des 3. Falles $\check{5} \mathrm{~mm}$ hoch.

25. III. Regenerat des 4 . Falles $4 \mathrm{~mm}$ hoch.

b) Gezackte Kämme: a) Triton cristatus Laur. - Das Männchen des großen Kammolches (Triton [Molge] cristatus Laurenti) trägt inter nuptias einen bis $15 \mathrm{~mm}$ hohen Kamm, dessen Rand aus tiefen, scharfen Zacken besteht, und, da letztere meist schief, caudalwärts geneigt sind, mit botanischem Fachausdrucke *gesägt * heißen müßte. Bisweilen ist jede einzelne Zacke abermals gesägt, der ganze Kamm also »doppelt gesägt . Der Kamm beginnt als niedrige Leiste schon zwischen den Augen, ist am Nacken ungefähr halbhoch und erreicht in der Rückenmitte seine volle Höhe, um dann in ziemlich steilem Bogen abzusinken und diesseits des Cloakenmeridianes ganz aufzuhören. Jenseits vom Cloakenmeridian beginnt der Kamm von neuem und erreicht als (selten gezackter, meist nur ziemlich tief ausgeschweifter) Schwanzsaum nochmals beträchtliche Breite (Fig. 4). Übrigens unterliegt die Kammformation bei Triton cristatus ziemlich unabhängig von den Subspecies und Varietäten dieser Art mannigfachen Abänderungen, unter welchen eine, bei der über der Cloakengegend keine Unterbrechung zu bemerken ist, sondern wo der Kamm vom Nacken zur Schwanzspitze in continuo und ohne bedeutende Höhenunterschiede dahinzieht, besonders erwähnt sei. 
Wird nun der Kamm seiner ganzen Länge nach entfernt, so wächst an seiner Stelle ein andrer, der gleich zu Beginn ebenso tiefbraun gefärbt ist wie der normale, aber am Rande nicht mehr "gesägt", sondern "kerbsägig * ist: die einzelnen Kerben sind klein and an der Spitze oval zugerundet (Fig. 4a). Zweifellos liegt hier eine im Vergleich zu den spitzen, scharfen, tiefen Zacken des primären Kammes hypotype Bildung vor. Der Schwanzsaum aber ist eher hypertypisch regeneriert zu nennen: denn war er vorher nur ansgeschweift, so ist er nun gleich dem Rückenkamm kerbsägig, so daß der regenerierte Rand seinem ganzen Verlaufe nach gleichmäßige Skulptur zeigt. Noch etwas ist bei der Totalregeneration des Kammes besonders auffällig: war primär uber der Cloakengegend eine Unterbrechung gewesen, so ist diese beim Regenerat sekundär überbrückt, während sich die neuerliche Bildung eines schon vorher ununterbrochen verlaufenen Kammes in dieser Beziehung mit der primären Bildung ubereinstimmend repräsentiert. Nimmt man die Kammolchform mit ununterbrochenem Kamm als die ältere, ursprünglichere an, was zum mindesten sehr nahe liegt, da bei keiner andern Molchspecies Europas eine völlig unterbrochene Gestaltung wiederkehrt, so wïrde man in der Bildung eines kontinuierlichen Saumes, wo ein diskontinuierlicher gestanden hat, einen Fall von atavistischer Regeneration erblicken müssen.

Einen sehr interessanten Verlauf nahmen die Variationen dieses Versuches. Wurde nur der Schwanzsaum entfernt, so beschränkte sich das Auftreten des kerbsägigen Randes auf das Regenerat, die übrigen Teile blieben gesägt, erfuhren aber eine kompensatorische Reduction insofern, als die Spitzen der einzelnen Zacken ilhre Schärfe verloren, sich abrundeten und die Zwischenräume der Zacken ebenso eine minder steile und minder tiefe, am Grunde minder eckige Formation békamen. Wurde nur der Ruickenteil des Kammes entfernt, so verliert der Schwanzsaum seine Ausbuchtungen und wird ganzrandig (Fig. 4b). War der Kamm anfänglich doppelt gesägt gewesen, so machte das für die regenerierten Partien insofern keinen Unterschied, als sie trotzdem einfach kerbsägig ausfielen; auf den unoperiert gebliebenen Partien aber waren die auf den großen Zacken stehenden kleinen gänzlich eingeschmolzen worden, die großen nebstbei noch in geschilderter Weise abgerundet und verflacht.

Wurden jedoch zwei distale Schwanzdrittel oder die Schwanzhälfte amputiert, so regenerierten diese mit einem kurze Zeit, besonders gegen die Spitze zu, ganzrandigen, dann sehr klein kerb- 
sägigen Rand; die Rückdifferenzierung greift aber unabgeschwächt auf das nicht amputierte Drittel bzw. die nicht amputierte Hälfte des Schwanzes iber und auch die Zacken des Rückenkammes verlieren noch mehr als in dem fruberen Falle ihre Schärfe, zeigen mehr und weiter vorschreitende Tendenz, ihre Spitzen und Zwischenräume abzurunden und auszuebnen (Fig. 4c). Die kompensatorische Wirkung ist an Kämmen, die urspringlich schon in continuo verlaufen waren, eine entschieden stärkere als an solchen, die uber der Cloake unterbrochen gewesen waren, was sich ungezwungen aus der leichteren Gewebsverbindung im ersten Falle erklärt gegentiber der Vermittlungsnotwendigkeit von seiten der kammlosen Rückenhautstrecke im zweiten Falle.

All diese kompensatorischen Vorgänge sind nicht zu verwechseln mit einem SchrumpfungsprozeB des Kammes: denn erstens konnte der Kamm während seiner Umwandlung am Rande, anch wenn diese Umwandlung eine Vereinfachung war, an Höhe noch zunehmen; zweitens sind die Zacken schon eines ganz niedrigen, gleichviel ob im Erheben oder im Zurïckgehen begriffenen Primärkammes scharfspitzig, wenn auch noch klein.

\section{Auszug aus dem Versuchsprotokoll:}

1907. 1. IV. Abschneiden des gesägten Triton cristatus-Kammes seiner ganzen Länge nach, nur auf dem Rücken, nur auf dem Schwanze und Amputation von $2 / 3$ des Schwanzes bei je 10 of 0 .

6., 5., 5. IV. Auftreten eines normalfarbigen, kerbsägigen Saumes im 1., 2. und 3. Falle.

16. IV. Maximalstand der kompensatorischen Reduction nicht operierter Kammpartien im 2. und 3. Falle erreicht.

19. IV. Regeneration eines ganzrandigen Saumes anf dem Schwanzregenerat fast zugleich mit dessen Erscheinen einsetzend, im 4. Falle.

20. V. Maximalstand der kompensatorischen Reduction nicht operierter Kammpartien in diesem (4.) Falle erreicht.

B) Triton vulgaris L. - Da von der Art Triton [Molge] vulgaris Linné (dem kleinen Teichmolch) gegenwärtig mehrere Unterarten und Varietäten unterschieden werden, ist es zunächst notwendig, zu bemerken, daß die im folgenden beschriebenen Operationen, soweit nicht ausdruicklich anders angegeben, an der typischen Form derselben (subsp. typica Wolt.) ausgeführt sind.

Das Männchen dieser Unterart trägt zur Laichzeit einen 3 bis $5 \mathrm{~mm}$ hohen, buchtig gezähnten, sim Nacken beginnenden und uber dem After nicht unterbrochenen, vielmehr dort besonders hohen Kamm\& (De Bedriaga, S. 383). Auf Taf. III Fig. 5 ist dieser Kamm in 
seinem Normalzastande abgebildet. Schneidet man ihn seiner ganzen Länge nach weg, so wächst binnen 4 bis 5 Tagen, und zwar gleichschnell, ob man den Schnitt dicht am Rumpf oder höher, etwa in halber Kammböhe, geführt hat, an seiner Stelle ein vollkommen ganzrandiges, aber an Höhe hinter dem primären Kamm nicht zurtickstehendes Regenerat. Nicht nur in seinen Konturen, sondern auch in seiner Färbung und Zeichnung ist jenes Regenerat minder differenziert als der ursprügliche Kamm: dieser ist gleich dem Rumpf mit rundlichen, meist ziemlich scharf umgrenzten, dunkelbraunen bis schwarzen, manchmal olivengrünen Punkten geziert, der regenerierte Kamm zeigt nur halb erloschene, verschwommene, hell olivbraune Flecken. Die in Gestalt und Farbmerkmalen sich aussprechende Hypotypie macht auch nicht so bald der primären Form Platz. Ich sah Teichmolche mit derartigen Regeneraten ein volles Fribjahr einhergehen; erst in der nächstfolgenden Brunftperiode entsteht wieder ein gezähnter Kamm, aber mit weniger und schwächeren Zacken als ehedem.

Schneidet man nur den Rückenkamm oder nur den dorsalen Schwanzflossensaum allein weg, so regeneriert der betreffende Abschnitt ganzrandig (Fig. $5 a$ und $5 c$ auf Taf. III) außerdem aber greifen regulatorische Prozesse auf den stehengelassenen Teil des Kammes über: die Zackenzahl vermindert sich, ja kann bis auf einzelne, als schwache, stumpfe Vorsprïnge erhalten bleibende Zacken ganzrandig werden. Am schwächsten ist dieser korrelative Einfluß bei Wegnabme einer dorsalen Kammpartie auf den ventralen Teil des Schwanzflossensaumes: Entfernung des Rückenkammes bleibt dort ganz ohne EinfluB, Entfernung des dorsalen Schwanzflossensaumes läßt die Zackenzahl der ventralen Partie etwas, aber nicht viel geringer werden (z. B. Verminderung von acht auf fünf, in einem andern Falle von neun auf sieben Zacken). Entfernt man umgekehrt nur den ventralen Schwanzsaum, so ubt sein Regenerationsprozeß auf den Rückenkamm gar keinen Einfluß ans und bewirkt im dorsalen Schwanzsaum nur die Einschmelzung relativ weniger Zacken (zwei beobachtete Beispiele: Reduction von elf auf acht, von zehn auf acht Zacken, wobei ich als Grenze zwischen Ruicken- und Schwanzsaum eine zum candalen Cloakenrand hinziehend gedachte senkrechte Linie annahm). Der korrelative Einfluß ist also in antero-posteriorer Richtung bedeutend stärker als in dorso-ventraler: man braucht nur ein $10 \mathrm{~mm}$ langes Stückchen nahe der Schwanzspitze aus dem Saum herauszuschneiden, so findet noch ganz vorn am Genick Zackenreduction statt. 
Noch viel intensiver gestaltet sich dieser kompensatorische Vorgang, wenn man den Triton vulgaris-Männchen ganze Schwanzteile ampntiert, also sie nicht bloß des Flossensaumes, sondern auch zugleich der Muskel- und Skeletpartie beraubt. Amputiert man ein distales Drittel, so wird der ganze übriggebliebene Schwanzsaum glatt ganzrandig, der des Rückenkammes schmilzt am Rande zu sanft gewellten Ausrandungen ein. Amputiert man die Hälfte oder zwei Drittel, so wird auch der ganze Ruickenkamm ganzrandig und bleibt es noch, bis das Regenerat der verlorenen Schwanzteile vollendet ist; dann erst kommen langsam wieder kleine Zacken zum Vorschein (Fig. 5b).

Mehrere südliche Unterarten von Triton vulgaris, so die subsp. meridionalis Blgr. und die subsp. graeca Wolt., haben einen ganzrandigen und allerdings anch viel niedrigeren, nur 2-4 mm hohen Vertebralflossensaum an Stelle des Zackensaumes der forma typica. Man wäre vielleicht geneigt, daraus, daß der regenerierte Vertebralsaum der letzteren ebenfalls ganzrandig ist, auf ihre Abstammung von einer jener stidlichen Unterarten zu schließen, eine Ansicht, die ohnedies mit andrer Motivierung bereits von MÉHely (S. 281 oben) ansgesprochen worden ist. Man würde daher eine atavistische Regeneration konstatieren. Ich glaube, daß ein solcher Schluß in diesem Falle und auf Grund der bis jetzt gewonnenen Ergebnisse noch nicht zulässig wäre. Denn erstens kann es sich um eine bloße Hemmungsbildung handeln, zweitens haben die Molchlarven samt und sonders ganzrandige Flossensäume, so daß die Wied erholung eines ontogenetischen Stadiums vorliegen kann und noch nicht die eines phylogenetischen vorzuliegen braucht. Drittens wächst auch der regenerierte ganzrandige Kamm von Triton vulgaris typicus während der Regeneration zu einer Höhe an, welche der des nicht regenerierten primären Kammes fast gleichkommt, die er aber bei subsp. graeca und meridionalis, soweit bekannt, niemals erreicht.

Um in diese Frage Klarheit za bringen - obwohl es von vornherein wenig wahrscheinlich war, $\mathrm{daB}$ es auf diese Weise gelingen werde - , stand mir noch ein reiches Material brünftiger Männchen der beiden genannten, südlichen Vulgaris-Formen zugebote, an deren ganzrandigen Kämmen ich zur Kontrolle die analogen Operationen ausfuhrte, wie sie schon ron Triton vulgaris typicus beschrieben wurden. Es wäre ja daran zu denken gewesen, daß die Regenerate zackig oder kerbig ausfallen würden und dadurch im Gegensatz zu der früher angedeuteten Ansicht auf subsp. typica als Stammform hingewiesen hätten. Es war aber nicht der Fall: ich erhielt in allen 
Kombinationen von Triton vulgaris meridionalis und graeca ganzrandige Flossensaum-Regenerate mit der Erscheinung der hypertrophischen Regeneration wie bei Triton alpestris. Die nachgewachsenen ganzrandigen Kämme der studlichen Teichmolche erreichten jetzt mit 3 bis $4 \frac{1}{2} \mathrm{~mm}$ beinahe die Durchschnittshöhe der nachgewachsenen ganzrandigen Kämme der typischen Teichmolche, - ein sicherer Schluß auf die Natur dieser Kämme als Atavismen ist somit nicht möglich.

Die Neigung niedriger, ganzrandiger Kämme, hypertrophisch zu regenerieren, veranlaßte mich dazu, die Vertebralleiste, durch welche das briuftige Weibchen von Triton vulgaris ausgezeichnet ist und die namentlich bei Triton vulgaris meridionalis sich mit ihren $1 \frac{1}{2}$ bis $2 \mathrm{~mm}$ Höhe schon ganz gut zur Operation eignet, ihrem ganzen Verlaufe nach abzuschneiden, um allenfalls nach erfolgter hypertrophischer Regeneration Weibchen mit Kämmen zu erhalten, die an Höhe oder gar auch in bezug anf Konturierung denen der Männchen gleichkommen. Bisher erzielte ich aber in dieser Richtung kein Resultat: die Vertebralleiste des Weibchens regeneriert ebenso gut und schnell wie der Kamm des Männchens, aber wird nicht höber, als sie es zuvor war.

Auszug aus dem Versuchsprotokoll:

Serie mit Schwanzamputationen.

1906. 22. IV. Amputation ron 1/3, 1/2, $2_{3}$ des Triton vulgaris-Schwanzes bei je 12 o \& der forma typica.

10., 8., 7. V. Anfang der Schwanzregeneration in Form einer halb eiförmigen Kuppe im 1., 2., 3. Falle.

16., 15., 15. V. Beginn des Kammwachstums auf den Regeneraten in Form einer ganzrandigen Leiste im 1., 2., 3. Falle.

20. V. Gesamtlänge des Schwanzes infolge ungleich raschen Wachstums der Regenerate überall 14-15 mm bei durchschnittlich $69 \mathrm{~mm}$ Gesamtkörperlänge; Schwanzkamm im 1. Falle gewellt, im 2. und 3. Falle ganzrandig, Höhe $3,3,4 \mathrm{~mm}$.

1907. 5. V. Riickenkiimme bei allen überlebenden Exemplaren $(7,5,8)$ des 1., 2., 3. Falles an der höchsten Stelle 4-4,5 mm hoch, immer noch beinalle ganzrandig, doch mit beginnender Zähnelung im 2. und 3, bereits schwach gekerbt im 1. Falle.

Serie mit Amputationen verschiedener Kammpartien.

1906. 20. IV. Abschneiden des gezähnten Kammes von Triton vulgaris f. typ. seiner ganzen Länge nach, nur auf dem Riicken, nur auf dem dorsalen, nur auf dem ventralen Schwanzsaum bei je 12 ơ

24., 23., 23., 25. IV. Regeneration eines ganzrandigen, blassen Saumes im 1., 2, 3., 4. Falle.

28. IV. Auch der Schwanzkamm im 2, der Riickenkamm im 3. Falle 
ganzrandig geworden, mit Ausnahme von 1-2 Zacken ganz hinten im 2., 1-3 Zacken ganz vorn im 3. Falle.

30. IV. Verminderung der Zackenzahl des ventralen Schwanzsaumes von 8 auf 5 oder 9 auf 7 im 3., des dorsalen Schwanzsaumes von 11 auf 8 oder 10 auf 8 im 4 . Falle.

\section{Serie mit Kammamputationen an Tr. vulg. meridionalis} und graeca.

1907. 1. IV. Abschneiden des ganzrandigen, 2-3 mm hohen Kammes seiner ganzen Lïnge nach, nur auf dem Rückeu, nur auf dem Schwanze und Amputation der distalen Schwanzhälfte bei je 10 of $\$$ ron meridionalis and je 10 von graeca.

5., 5., 6., 15. IV. Regeneration eines ganzrandigen, farblosen Saumes im 1., 2:, 3., 4. Falle (hier auf dem Regenerat der distalen Schwanzhälfte).

10., 9., 9. IV., 1. VIII. Regenerate vollendet, d. h. gleich aussehend wie die entsprechenden primären Teile, in den 4 Fällen.

1. V. Die neuen Kämme des 1., 2. und 3. Falles (im 2. und 3. Falle einschl. der unverletzt gebliebenen Teile) höher als der frübere Kamm, 3-41/2 $\mathrm{mm}$ hoch.

Serie der Weibchen mit amputierten Vertebralleisten.

1907. 1. IV. Abschneiden der $1 \frac{1}{2} \mathrm{~mm}$ hohen, ganzrandigen Leiste ihrem ganzen Verlauf nach bei je 15 \&ᄋ von meridionalis und graeca.

4.-5.IV. Regeneration der Leiste in ihrer ursprünglichen Höhe.

2. Andre Hautwucherungen der Wassermolche.

a) Der Hautsaum an der Oberlippe von Triton cristatus: Für die Prüfung der Regenerationsfähigkeit des Labiallappens eignet sich der männliche Triton cristatus am besten, weil diese wenig auffällige und wenig beachtete Hautfalte hier während der Brunftzeit relativ am stärksten ausgebildet ist.

Schneidet man sie ringsum dicht am Lippenrande weg, so ist sie schon binnen 3 Tagen wieder nachgewachsen; zuerst ist sie, wie die meisten Wundheilgewebe bei Amphibien, fast reinweil, was der Physiognomie der Molche ein eigentümliches Gepräge verleiht, dunkelt aber im Laufe einer Woche vollständig nach.

Da, wie schon Spallanzani festgestellt und Werber neuerdings bestätigt hat, bei Triton auch die Kiefer regenerieren, so operierte ich den Labiallappen auch mitsamt den tiefer liegenden Gewebsteilen, auf denen er inseriert, d. h. ich schnitt das betreffende Stïck des Oberkiefers heraus. Der Regenerationsprozeß nahm etwa 6 Wochen in Anspruch, der Labiallappen war aber ausgeblieben; erst in der nächstjährigen Brunftperiode wurde er, und zwar in seiner früheren Farbe und Ausdehnung, wieder sichtbar. 
Auszug aus dem Versuchsprotokoll:

1906. 7. III. Labiallappen dicht an der Oberlippe (1. Serie), samt dem Oberkiefer (2. Serie) abgeschnitten bei je $15 \mathrm{Tr}$. cristatus - $§$.

10. III. Labiallappen in der 1 . Serie regeneriert, weiß.

16.-17. III. Regenerierter Labiallappen nachgedunkelt.

20.-24. 1V. In der 2. Serie der Oberkiefer (ohne Labiallappen) regeneriert. 1907. 3. III. Labiallappen am regenerierten Oberkiefer wieder aufgetreten.

b) Die Lappen an den Hinterbeinen von Triton vulgaris: Das Männchen des Triton vulgaris, subsp. typica, besitzt, solange es sich im Wasser aufhält, besonders aber nuptiarum temporibus, an den Hinterextremitäten stark abgeplattete Zehen, die von seicht ausgeschweiften Lappensäumen umgeben sind (Fig. 6).

Schneidet man rings um die Zehen nur jene Hautlappen weg, so treten schon nach 2 bis 3 Tagen neve von gleichem Aussehen an ihre Stelle; schneidet man jedoch die ganzen Zehen mit ab, so ist das Regenerat erst nach 2 bis 3 Wochen fertig: die Säume sind dann nicht mehr gewellt, sondern ganzrandig (Fig. 6a). Schneidet man eine Phalange nur teilweise, etwa die zwei Endglieder ab, so regenerieren diese binnen 8 bis 10 Tagen mit ganzrandigem Lappen; die Reduction der Ausbuchtungen ist aber kompensatorisch auf den ganzen Zehenlappen, auch auf die stehengebliebenen Teile, übergetreten. Schneidet man nur einige von den füf Hinterzehen, jene aber ganz $a b$, so erstreckt sich die Ausebnung des vorher ausgeschweift gewesenen Lappenrandes auch auf die Nachbarzehen: bei Amputation der ersten oder fünften Zehe nur auf die eine, bei Amputation einer dazwischenliegenden Zehe auf beide, die rechte und die linke Nachbarzehe. Amputiert man mehr als eine Zehe, so unterliegen alle übrigen Zehen der kompensatorischen Reduction.

Amputiert man das ganze Hinterbein in der Mitte zwischen Ansatz und Knie, so regeneriert es binnen 4 bis 5 Wochen und trägt dann, auch wenn das Tier im Wasser bleibt, stielrunde (nicht verbreiterte) Zehen ohne Hautsäume; erst in der nächstfolgenden Brunftperiode kommen diese - samt Abflachung der ganzen Zehe - wieder zum Vorschein, und zwar dann in der ursprünglichen, ausgeschweiften Gestalt.

\section{Auszug aus dem Versuchsprotokoll:}

1906. 26. III. Amputation der ansgeschweiften Zehenlappen, der 2 Endglieder einer Phalange, der ganzen 1., der ganzen 2., der 1. +2 ., aller 5 Phalangen, des ganzen Hinterbeines bei je $10 \mathrm{Tr}$. vulgaris- $\lesssim \overrightarrow{0}$. 28.-29. III. Regeneration der Zehenlappen im 1. Falle (ausgeschweift).

1.-3. IV. Regeneration der 2 Endglieder einer Phalange samt Lappen 
(ganzrandig, Reduction auch auf die übrigen Glieder derselben Phalange ubergegangen!.

7. IV. Regeneration der 1., bzw. 2. Phalange mit Lappen (ganzrandig, Reduction auf die 2., bzw. 1. und 3. Zehe ubergegangen).

7.-8. IV. Regeneration der 1. +2 . Phalange mit Lappen (ganzrandig, Reduction auch auf die 3.-5. Phalange übergegangen).

10.-16. IV. Regeneration aller 5 Phalangen, mit Lappen (ganzrandig).

18.-23. IV. Regeneration des Hinterbeines ohne Lappen und mit nicht verbreiterten Zehen.

1907. 10. III. Verbreiterung der Zehen am regenerierten Hinterbein, Bildung der Lappen (auggeschweift).

c) Schwimmbäute an den Hinterzehen von Triton palmatus Schneid.: Das Männchen des Schweizer- oder Fadenmolches (Triton [Molge] palmatus Schneider) besitzt, solange es sich im Wasser aufhält, besonders aber nuptiarum temporibus an seinen Hinterzehen sbald mehr, bald weniger deutlich eingebuchtete, die Zehen bisweilen bis zu ihrer Spitze verbindende, regelrechte Schwimmhäute* (BEDRIAGA S. 418).

Bei Prüfung der Regenerationsverhältnisse dieses sekundären Geschlechtscharakters gelangte ich zu genau analogen Resultaten wie bei den Zehenlappen von Triton vulgaris: das Ausschneiden der Schwimmhäute allein wird binnen 2 bis 3 Tagen von neuerlicher Hervorbringung gleichgebildeter, also ebenfalls eingebuchteter Schwimmhänte gefolgt, die sogar, wenn sie sich vorher nicht bis an die Zehenspitzen erstreckten, jetzt stets bis zu diesen, also weiter reichen als vorher (bypertrophische Regeneration); das Entfernen von Zehenteilen und ganzer Zehen löst binnen 2 bis 3 Wochen deren Regeneration mit Schwimmhäuten aus, die der Ausrandungen entbehren und am Saume ganz glatt und gerade verlaufen. Das Entfernen nur einer Zehe, welche aus der Verbindungshaut herausgeschnitten und wobei diese also am Bein gelassen wird, bewirkt, daß die beiden nun auf jener Seite der Insertionsstelle entbehrenden, lose hängenden Hautstïcke, nachdem sie sich an die regenerierte Zehe wieder angeschlossen haben, einen ganzrandigen Saum statt des eingebuchteten bekommen. Das Entfernen von mehr als einer Zehe, gleichviel ob mit oder ohne Schwimmhäute, überträgt die Hypotypie auf die nebenliegenden Zehenintervalle. Amputierte Beine endlich regenerieren binnen einem Monat oder etwas darüber, erlangen aber erst nach Jahresfrist, vorausgesetzt daß die Männchen abermals brünftig werden, die Spannhäute zurück, jetzt aber mit den Ausbuchtungen, wie die primären sie aufwiesen. 


\section{Auszug aus dem Versuchsprotokoll:}

1906. 10. V. Ausschneiden der ausgebuchteten Spannhäute, Amputation zweier Endglieder der 3. Phalange, Ausschneiden der 4. Phalange ohne Spannhäate, Amputation der 4.+5. Phalange mit Spannhäuten und des gesamten Hinterbeines bei je 10 Triton palmatus- $\hat{\jmath} \vec{\delta}$. (Wo nicht ausdriicklich anders bemerkt, versteht sich beiderseits vorgenommene Operation.)

12.-13. IV. Regeneration der Spannhäute (ausgerandet).

24. IV. Regeneration der 2 Endglieder der 3. Phalange samt Spannbiuten (beiderseits ganzrandig).

1.-2. V. Regeneration der 4. Phalange, Zusammenheilung mit den nicht weggeschnittenen Spannhäuten, diese dabei ganzrandig geworden.

3.-5. V. Regeneration der 4.+5. Phalange (alle Spannhäute der Hinterbeine ganzrandig geworden).

9.-13. V. Regeneration des Hinterbeines ohne Spannhäute.

1907. 6. IV. Wiedererscheinen der Spannhäute (ausgerandet).

d) Endfäden am Schwanze kommen unter den meinen Untersuchungen zugänglichen Arten bei Triton palmatus, den stidlichen Unterarten des Triton vulgaris (meridionalis und graeca), ferner bei Triton [Pelonectes] Boscai Lataste und beim Karpatenmolch (Triton Montandoni Blgr.) vor. Wenngleich Andeutungen der Endfäden in Form von Zapfen oder selbst kurzen Fäden auch bei den hochzeitlichen Weibchen vorkommen, erreichen jene doch nur beim Männchen, und zwar dessen Wasser- und Hochzeitsform, ihre eigentliche stattliche Ausbildung. Bei Triton palmatus und vulgaris graeca handelt es sich um einen feinen, schwärzlichen Faden, der in einer Länge von $6 \mathrm{~mm}$ ans dem an der Ursprungsstelle des Fadens scharf abgesetzten, wie abgehackten Schwanzende hervorragt (Fig. 7). Bei $T r$. vulgaris meridionalis entspringt der Faden nicht ans einer stufenförmig abgesetzten Stelle, sondern geht ans einer langen, allmählichen Verschmälerung des Schwanzflossensaumes hervor. Bei Tr. Boscai-Männchen sind mir bis $3 \mathrm{~mm}$ lange, allmählich aus dem Schwanzflossensaum verdünnte Fäden untergekommen, bei Tr. Montandoni solche von doppelter Länge.

Das regenerative Verbalten dieser Endfäden ist ein so tubereinstimmendes, daB ich es nicht separat zu behandeln brauche. Wird der Faden allein abgeschnitten, so regeneriert er, vorausgesetzt, daß die Tiere im Wasser bleiben, binnen 4 bis 5 Tagen unverändert, oder er wird sogar um 1 bis $2 \mathrm{~mm}$ lïnger als er es vorher war. Wird ein distales Schwanzdrittel mit abgeschnitten, so kann das zunächst als gewöhnliche Spitze regenerierte Schwanzende noch im selben, sicher aber im nächstfolgenden Frïhjahr einen Faden hervortreiben. Wird 
die Schwanzhälfte oder mehr abgeschnitten, so regenerieren zunächst Schwanzenden von stumpf zugerundeter Gestalt, die auch nicht eine Spur von Fadenbildung und ihrer Ansatzstufe, wie sie bei Triton palmatus und vulgaris graeca normalerweise vorhanden, sehen lassen (Fig. 7a). Nach einigen Monaten erbält das abgerundete Schwanzende eine Spitze, die aber nicht anders aussieht als z. B. der normal zugespitzte Schwanz von Triton alpestris (Fig. 3). Mit der nächsten Brunftperiode kann dann das Schwanzende wieder einen Faden erzeugen, es ist aber nicht regelmäßig der Fall; soweit meine Erfahrungen reichen, scheint die Hypotypie hier eine bleibende sein zu können. So habe ich zwei Triton palmatus-Männchen im Besitz, die seit mehreren Jabren pünktlich im April brünftig werden. Seit ich sie ihre Schwänze habe regenerieren lassen, bekommen sie zwar vom zweiten Fruhjahr seit der Amputation an wiederum einen Endfaden, interessanterweise aber nicht mehr einen mit abgestufter Ansatzstelle, sondern einen, der in sanftem Bogen in den Schwanzsaum übergeht wie bei Triton vulgaris meridionalis (Fig. $7 b$ ). Da die Larve von Triton palmatus keinen Endfaden besitzt, bei Regeneration eines solchen von abweichender Form am geschlechtsreifen Molch somit keine Wiederholung eines ontogenetischen Stadiums vorliegen kann, so wäre hier an atavistische Regeneration zu denken. Leider aber ist das mir vorliegende Material, welches diese Erscheinung zeigt, auf jene zwei Exemplare beschränkt und daher zu gering, um bestimmtere Schlüsse zu erlauben.

\section{Auszug aus dem Versuchsprotokoll:}

1905. 27. III., 8., 12. IV., 1. V. Amputation des Endfadens, des distalen Schwanzdrittels, der Schwanzhälfte bei je 10 to 0 von $T r$. Montandoni (1. Datum), palmatus (2. Datum), vulgaris meridionalis (3. Datum', Boscai und vulg. graeca (4. Datum).

1., 13., 16. IV., 5., 6. V. Regeneration des Endfadens bei Montandoni, Palmatus, Vulg. meridionalis, Vulg. graeca, Boscai.

11., 22., 27. IV., 15., 17. V. Regeneration des distalen Schwanzdrittels bei den 5 aufgezählten Molchformen: 1 Montandoni, 2 Palmatus, 6 Vulg. merid., 1 Vulg. graeca mit Faden, sonst ohne Faden.

18., 30. IV., 9., 22.-23., 26. V. Regeneration der Schwanzhälfte bei den 5 Molchformen, Schwanzende stumpf abgerundet.

1. VIII. Schwanzende iberall zugespitzt.

1906. 28. II., 1. III., 18.-20. III., 24.-25. III. Schwanzende erhält einen Faden bei 2 Montandoni, 3 Palmatus, 5 Vulg. meridion., 4 Vulg. gracca, die uibrigen bleiben einfach zugespitzt, ohne Faden, selbst ohne Zäpfchen.

1907. 2.-5. IV. 2 Palmatus erhalten Endfäden von der Gestalt derjenigen bei normalen Vulgaris moridionalis. 
e) Die Halswarze des Triton [Cynops] pyrrhogaster Boie: Das Männchen des japanischen Feuerbauches (Triton pyrrhogaster Boie) bekommt zur Brunftzeit, die sich in unsern Becken von Ende April an abspielt und bis Ende Juni andauert, an beiden Halsseiten, dort, wo die Gularfalte lateral endigt und eine rinnenförmige Fortsetzung derselben, die nach hinten und oben zum Hinterrand der Parotis verläuft, ansetzt, eine warzenförmige, halbkugelige, driisige Auftreibung von Hanf- bis fast Erbsengröße $(2$ bis $31 / 2 \mathrm{~mm}$ Durchmesser), scharlachroter Farbe mit bläulich-violettem Reif und mehreren Drüsenöffnungen.

Schneidet man diese Warze glatt ab, was wegen ihres Drüsenreichtums nicht ohne ausgiebige Entleerung eines milchigen Saftes vonstatten geht, so wächst sie binnen 9 bis 10 Tagen wieder heran, indessen ohne die frühere Dicke wieder zu errreichen. Von ibrer Farbensattheit hat sie, wenigstens innerhalb der laufenden Brunftperiode, gleichfalls viel eingebüßt: sie zeigt sich nach der Regeneration schwarz verfärbt, wohl infolge der Umbildung ausgetretener Blutbestandteile in dunkles Pigment. Auch entbehrt die regenerierte Warze zunächst der Porenöffnungen: die Haut spannt sich ganz glatt und straff darïber, ohne Unterbrechungen ${ }^{1}$ ).

Trägt man die ganze umgebende Haut des Halses auf einer Strecke $a b$, deren Länge durch die Länge der Parotis gegeben erscheint, so heilt die Wunde binnen 2 Wochen oder noch etwas rascher und erzeugt regenerativ eine neue Hant, die der alten gleicht; die Warze aber bildet sich nicht mehr. Da ich diese Operation erst heuer ausgefuhrt, bleibt abzuwarten, ob die Warze mit Eintritt der nächstfolgenden Brunftperiode doch wieder entstehen wird.

\section{Auszug aus dem Versuchsprotokoll:}

1907. 29. IV. Amputation der roten Halswarze (1. Serie ; Abtragung der Halshaut samt Warze (2. Serie) bei je $6 \mathrm{Tr}$. pyrrhogaster-o 0 .

7.-8. V. Regeneration der Halswarze (1. Serie), verkleinert und schwarz. 11.-13. V. Regeneration der Halshaut (2. Serie) ohne Warzenbildang.

3. Der Sporn am Hinterbein von Triton rusconii Gené.

Der sardinische Wassermolch (Triton [Euproctus] rusconii Gené) besitzt im männlichen Geschlechte sehr charakteristische sekun-

1) Wie mir Freund WouterstonfF brieflich mitteilt, hat auch er Regeneration der Warze beobachtet an einem Männchen, welches auf jener ein Geschwür bekam. Die Warze wurde deshalb abgeschnitten, worauf die Wunde verheilte und die Warze sich neuerdings bildete. 
däre Sexualcharaktere, welche auch außerhalb der Brunftperiode erhalten bleiben und sich deswegen zu einem bequemen Untersuehungsobjekt für Regenerationszwecke gestalten würden, wenn diese Molchart nicht gegen Verletzungen viel empfindlicher wäre als ihre bisher besprochenen Verwandten. Mehrmals mußte ich frisches Material beziehen, was nicht immer leicht und stets mit ziemlich erheblichen Kosten verbunden ist, ehe ich an einem geringen Prozentsatz der operierten Tiere positive Resultate erlangte. Diese Empfindlichkeit steht in schroffstem Gegensatz zur Widerstandsfähigkeit der übrigen von mir behandelten Tritonen, bei denen nicht viel tiber $1 \%$ an den Folgen der Operation zugrunde ging.

Das erste der männlichen Attribute von Triton rusconii besteht in einem Sporn, der nahe der Fußwurzel, an der Hinterkante des Unterschenkels hervorragt. Nach De BedriagA (S. 688) verdankt dieser Sporn einer exzessiven Entwicklung des Processus styloideus fibulae, bzw. einer auf dem letztern aufsitzenden Knorpelkappe seine Entstehung. Distalwärts vor ihm liegen noch zwei bis drei kleine Höckerchen, welche der Außenkante der Fußwurzel ein kammartiges Aussehen verleihen (Fig. 8). Das zweite männliche Attribut beruht in der hechtartig verlängerten, abgestutzten Schnauze nebst a ufgetriebenen Backen, während der Kopf des Weibchens ungefähr das Aussehen eines typischen Triton-Kopfes bewahrt hat.

Beide Bildungen unterzog ich operativen Eingriffen, von denen aber die an den Kiefern, wie ich gleich von vornherein bemerken muß, bis jetzt ein durchaus negatives Erge bnis hatten, da die Tiere immer wieder, so oft ich auch den Versuch ernenerte, eingingen, während doch Triton cristatus und alpestris, jedenfalls auch die andern gewöhnlichen, daraufhin noch nicht untersuchten Tritonarten, der Kieferamputation ohne weiteres gewachsen sind.

Hingegen erhielt ich nach Amputation der Hinterbeine endlich Regenerate. Durch die Güte des Herrn Alois EgGer in Linz, der mir am 8. Juli 1905 sieben von ihm selbstgezüchtete; damals $1 \mathrm{Jahr}$ alte Larven ibermittelte, kam ich in die glückliche Lage, meine Versuche an solchen, die ja ein viel größeres Regenerationsvermögen besitzen mußten als arterwachsene Tiere, beginnen zu dürfen. An den Larven sind die beschriebenen Sexualcharaktere nicht zu unterscheiden, so daß ich wie bei den Froschquappen auf gut Gluick operieren mußte, doch war zu hoffen, daß unter den sieben Larven sich auch einige männliche befinden würden. Letzteres traf in der Tat zu, wie sich schon nach einem halben Jahre zeigte, indem an nicht 
weniger als sechs erst kurze Zeit metamorphosierten Exemplaren an der Stelle, wo der Sporn stehen soll, ein kleines Höckerchen, fast zugleich damit auch an der Stelle, wo die kammartigen Erhebungen der Fußwurzel-Außenkante erwartet wurden, zwei weitere, noch kleinere Höcker zum Vorschein kamen. Nach einem weiteren Jahr war die typische Bildung des Spornes und der von ihm distalwärts gelegenen Unebenheiten vollendet.

Diese Beschreibung bezieht sich auf den normalen Entwicklungsgang nicht operierter Hinterbeine, denn ich hatte meinen Larven nur das eine - rechte Hinterbein - amputiert. Sein Regenerat war 6 Wochen nach der Operation so groß geworden, daß man es nicht mehr vom Hinterbein der Gegenseite unterscheiden konnte, vermochte aber zu dieser Zeit natürlich noch keine Anlage des Spornes aufzuweisen, da eine solche damals auch noch dem unverletzten Beine fehlte. Nur wenig später als an diesem erschien der kleine Sporn nebst den übrigen Unebenheiten am Regenerat. Um dieselbe Zeit ist ferner auch schon eine geringe Streckung der Kiefer bei den Männchen zu bemerken, deren sekundäre Geschlechtsattribute somit gut $1^{1 / 4}$ Jahr vor Erreichung der Geschlechtsreife auftauchen.

Die Amputation der Hinterbeine an geschlechtsreifen Männchen erzielte ebenfalls keine dauernde Hypotypie. Das Regenerat, welches binnen durchschnittlich 10 Wochen soweit fertiggestellt war, daß die fünf. Phalangen differenziert erschienen, bekommt nach weiteren 2 bis 3 Wochen mehrere leichte tuberkelartige Anschwellungen in der Gegend des Processus styloideus fibulae, die allmählich die charakteristische Gestalt des Spornes und seiner Nebenhöcker gewinnen (Fig. 8a) und sich bei direkter Präparation auch anatomisch durchaus als solche erweisen.

\section{Auszug aus dem Versuchsprotokoll:}

Serie der Larven.

1905. 10. VII. Amputation des rechten Hinterbeines an 7 ein Jahr alten, fast verwandlungsreifen Larven.

25. VIII.-1. IX. Rechtes Hinterbein vollständig regeneriert.

27. XII. Metamorphose.

1906. 18. I. Das linke, unverletzt gebliebene Hinterbein erhält bei 6 Exemplaren die Anlage der Spornbildung.

10. II. Das Regenerat erhält ein kleines Höckerchen in der Gegend des Processus styloideus fibulae.

15. II. Distalwirts davon 2 weitere Höckerchen entstanden.

1907. März. Der proximalste der drei Hücker ist zum typischen Sporn ausgewachsen. 
Serie der geschlechtsreifen Männehen.

1905. 2. VII. Amputation des rechten Hinterbeines an 10 geschlechtsreifen $\delta \hat{\delta}$. 16. VIII. Hinterbein regeneriert bei 3 Exemplaren, die übrigen tot.

1.-9. IX. Auftreten mehrerer Unebenheiten an der Außenkante des Unterschenkels.

1906. 20. III. Die Unebenheiten haben sich in den Sporn mit seinen Nebenhöckern differenziert.

4. Sekundär sexuelle Farbcharaktere bei Triton cristatus.

a) Der blauweiße Schwanzstreifen des Männchens: Solange das Männchen des Kammolches (Triton cristatus) im Wasser lebt, am meisten natürlich wiederum inter nuptias, sind seine Schwanzseiten mit einer 3 bis $4 \mathrm{~mm}$ breiten, silberweißen, meist perlmutterartig blauschillernden Binde geziert (Fig. 4).

Auch hier ist wiederum eine $\mathrm{zw}$ e if ache Operation möglich, um die Regenerationsfähigkeit dieses Merkmals auf die Probe zu stellen: Abtragen der Haut von den Flanken des Schwanzes und Amputation des Schwanzes. Zunächst fübrten beide Operationen zu einem hypotypen Resultat: der Schwanzstreifen erschien nicht wieder, sondern das neue Integument hatte die gleichförmig dunkelbraune, durch einzelne schwärzliche Flecken verdüsterte Farbe des übrigen Schwanzes and Körpers.

Während aber an jenen Kammolchschwänzen, die nur Hautdefekte erlitten hatten, der silbrige Streifen mit dem Eintritt der nächstfolgenden Laichperiode wiederum auftrat (Fig. 4b), war dies bei denjenigen Molchen, die auch eine Restitution der übrigen Gewebe geleistet hatten, nicht der Fall. Sie wurden brïnftig, befruchteten ihre Weibchen, waren im Vollbesitze des Kammes und des nuptialen Oberlippensaumes, der Schwanz aber entbehrte der leuchtenden Hochzeitsfarben (Fig. 4c).

Wurde nur ein relativ kleiner Teil des Schwanzes amputiert, so daß am restlichen Stummel ein Teil der Flankenbinde intakt blieb, so verschwand dennoch auch dieser Teil im Verlaufe der Regeneration, wurde zuerst verschwommen, undeutlich konturiert und durch dunkleren Anflug verunreinigt, um endlich ganz der braunen Grundfarbe Platz zu machen. Bei Ausheilung partieller Hautdefekte hingegen, an denen der Streifen beteiligt ist, werden zurückgebliebene Teile des letzteren nicht eingezogen; nur an der Wundbegrenzung mischt sich etwas Braun in das leuchtende Blauweiß, ohne aber dieses ganz zum Schwund zu bringen. 
Auszug aus dem Versuchsprotokoll:

Serie mit bloßen Hautdefekten.

1906. 8. IV. Abtragen der Haut von der rechten Schwanzflanke in der proximalen Hälfte des weißen Längsstreifens und in seiner ganzen Ausdehnung bei je 10 ô $\hat{o}$ von Triton cristatus.

19.-23. IV. Die neue Haut über der kleineren Wunde fertig, von brauner Farbe, an der Wundgrenze auch der intakt gebliebene Streifenteil etwas verduistert.

27. IV.-1. V. Die nene Haut über der großen Wande fertig, durchwegs braun und von der iibrigen Körperfarbe nicht zu unterscheiden.

1907. 5. IV. Silberweiße Binde in beiden Fällen wieder erschienen.

Serie mit Schwanzamputationen.

1906. 8. IV. Amputation von ${ }_{1 / 3}, 2 / 3$ des Schwanzes bei je $10 \delta \delta$ von Triton cristatus.

1. V. Regenerat des eines Schwanzdrittels fertig, einfarbig braun, ohne Streifen.

8.-9. V. Streifen auch auf den stehengebliebenen $2 / 3$ dieses Schwanzes erloschen.

7. V. Regeneration der zwoi am 8. IV. amputierten Schwanzdrittel fertig, einfarbig braun.

1907. April. Silberweiße Binde in beiden Fällen trotz eingetretener Brunft nicht wieder erschienen.

b) Die gelbe Vertebrallinie des Weibchens: Das Weibchen des Kammolches besitzt häufig, namentlich in der bei Wien häufiger als die typische Form vorkommenden subsp. carnifex Laur., längs der vertieften Rückenmitte eine im Nacken beginnende schwefeloder ockergelbe Linie. Bei älteren Weibchen der typischen Form sind von dieser Linie meist nur geringfiggige Reste vorhanden, auch bei der subsp. carnifex ist sie manchmal undeutlich. Bei jungen Tieren hingegen erfreut sie sich einer allgemeinen Verbreitung und grellen Färbung. Am intensirsten ist natürlich die Vertebrallinie, wo sie sich bei geschlechtsreifen Weibchen erhalten hat, wie alle Zeichnungselemente in der Paarungszeit nuanciert, aber wenn iberhaupt, auch außerhalb derselben vorhanden.

Dieser labile Charakter der gelben Rückenmedianlinie ließ mich erwarten, daß sie nach Regeneration des betreffenden Hautstreifens, den ich mit Hilfe eines schmalen, sehr scharfen Skalpels den zuvor in Äther narkotisierten Tieren abtrug, gar nicht mehr erscheinen werde. Es kam aber anders.

Die über der Wunde neu ausgespannte Haut hatte zu allernächst die weißliche Farbe, welche das Narbengewebe der Amphibien überhaupt aufzuweisen pflegt. Die Ränder färbten sich bald bräunlich, die Mitte abèr blieb hell, färbte sich dann gelblich und endlich 
scharf schwefelgelb bis rotgelb, auch bei solchen Weibchen, die primär eine sehr wenig ausgeprägte Medianlinie aufzuweisen gehabt hatten.

Nun glaubte ich den Regenerationsproze $B$ beendigt, was aber nicht der Fall war. Die bräunliche Tönung der ehemaligen Wundränder zu beiden Seiten der gelben Längslinie verduisterte sich zu Dunkelbraun, der Farbe des übrigen Rückens. Diese dunkle Farbe griff nun aber auf das schmale gelbe Mittelfeld über und brachte es - abgesehen von geringen Resten, die am Nacken mancher Exemplare erhalten blieben -- ausnahmslos vollständig zum Schwund.

Der beschriebene Regenerationsablanf ist deshalb im hohen Grade bemerkenswert, weil alle jungen, frischverwandelten Weibchen, wie schon bemerkt, mit großer Regelmäßigkeit sowohl in der subsp. typica als auch in der subsp. carnifex den gelben Rückenlängsstreifen aufweisen, selbst dann, wenn die geschlechtsreifen Tiere seiner entraten. Somit scheint der RestitutionsprozeB, trotzdem er nur einen Hantdefekt betrifft und keine geformte morphologische Einheit, streng: mit der ontogenetischen Entwicklung parallel gelaufen zu sein.

Auszug aus dem Versuchsprotokoll:

1906. 10. III. Abtragen der Haut von der Vertebrallinie bei 10 Triton cristatus $c \subseteq$ mit deutlichem und $10 \subsetneq \&$ mit kaum vorhandenem gelben Streifen.

22.-25. III. Über der Wunde neue Haut gebildet, weiß.

30. III.-4. IV. Mittellinie dieses Hautstreifens schwefelgelb, Seitenpartie bräunlich verfürbt.

10. IV. Seitenpartie dunkelbraun verfärbt, im allgemeinen von der lebhaft gelben Mittellinie scharf abgehoben, an einigen Stellen auf sie übergreifend.

21.-26. IV. Mittellinie hat bis auf Nackenteile, die bei $9 \circ 0$ Reste davon bewahren, das Dunkelbraun des übrigen Kückens angenommen.

1907. Frühling. Gelb ist auf der Mittellinie trotz eingetretener Brunft nicht wieder erschienen.

\section{Zusammenfassung.}

\section{A. Regenerationen.}

Typiscbe Regenerationen (ohne Einschiebung provisorischer Hypo- oder Hypertypie bis zur Erlangung der typischen Gestalt) liefern die männlichen Geschlechtsattribute an den Gliedmaßen der Froschlurche; ferner der Sporn am Hinterbein von Triton ruscomii; was ihre Form anlangt, die ganzrandigen Kämme des Triton alpestris- 
Männchens und marmoratus-Männchens, vulgaris-Weibchens, vulgaris meridionalis- und graeca-Männchens, weiters die Schwanzfäden mancher Tritonarten, falls nicht mehr als ein Drittel des Schwanzes mit abgeschnitten wird; die Labiallappen der brïnftigen Tritonmännchen, falls der Kiefer bei der Operation intakt blieb; die Zehenlappen des männlichen Triton vulgaris, falls die Zehen intakt blieben; und endlich unter derselben Bedingung die Schwimmbäute des männlichen Triton palmatus.

Hyp otypische Regenerationen liefern provisoriseh der Kehlstimmsack von $H y l a$, wenn an geschlechtsreifen Männchen operiert; die Labiallappen der Tritonen, falls mit dem Kiefer operiert; die Zehenlappen von Triton vulgaris und Schwimmhänte von Triton palmatus, falls mit Zehengliedern, ganzen Zehen oder Gliedmaßen amputiert; was ihre Farbe anlangt, die ganzrandigen Tritonkämme; auch was ihre Form anlangt, die gesägten und gezähnten Tritonkämme; endlich die blauweiße Schwanzbinde des männlichen Triton cristatus, wenn nichts als der betreffende Hautstreifen abpräpariert worden war. Definitive Hypotypie scheint beim Doppelstimmsack von Rana esculenta; bei den Schwanzfäden der Tritonen, falls jene mit mehr als einem Drittel des Schwanzes abgeschnitten wurden; bei der Halswarze des männlichen Triton pyrrhogaster; der Schwanzbinde des männlichen Triton cristatus, wenn gleichzeitig Teile des Schwanzes zu restituieren waren; und der Vertebrallinie des weiblichen Tritore cristatus (hier nach provisorischer Verstärkung) einzutreten.

Hypertypische Regenerationen liefern: der fast ganzrandige Kamm von Triton blasii (regeneriert feinkerbig); der ausgeschweifte Schwanzsaum von Triton cristatus, falls die Muskel- und Skeletpartie des Schwanzes intakt blieb (regeneriert kerbsägig).

Hypertrophische Regeneration kommt zuweilen vor bei den Kämmen der männlichen Triton alpestris, vulgaris meridionalis und vulgaris graeca, den Schwimmbäuten von Triton palmatus und den Schwanzfäden der zuletztgenannten Art wie auch mehrerer andrer Tritonspecies.

Als Regeneration mit Wiederholung ontogenetischer Stadien ist ein großer Teil der hypotypischen Regenerationen anfzufassen: so der weiß und glatt statt braun und faltig regenerierte Stimmsack von Hyla, der ganzrandig statt gezähnt regenerierte Kamm von Triton vulgaris typicus. Ferner ist das anfangs schärfere Vortreten der gelben Vertebrallinie des Triton cristatus-Weibchens hierher zu stellen. 
Als Regeneration mit Wiederholung phylogenetischer Stadien könnte das hypertypische Regenerat des Triton blasiiKammes, das ununterbrochen verlaufende Regenerat des vorber iber der Cloake eingesattelten Triton cristatus-Kammes und das allmählich in den Schwanzsaum übergehende Regenerat des vorher staffelförmigen Endfadens von Triton palmatus aufgefaßt werden.

Die Detailbefunde sind die folgenden:

1) An Extremitäten der Froschlurche, die während der Larvenperiode regeneriert sind, bilden sich beim Eintritte der Geschlechtsreife und ersten Brunft alle das Männchen auszeichnenden Sexualcharaktere in typischer Weise aus:

a) an der Vorderextremität die einwärts gedrehte Stellung des muskelstarken Armes; die Verdickung des Daumens und der Daumenballen; die Zehen- und (bei Bombinator) die Armschwielen.

b) an der Hinterextremität von Bombinator pachypus ebenso die dort vorhandenen Zehenschwielen.

2) Die Schallblasen der männlichen Froschlurche sind ebenfalls regenerationsfähig:

a) der einfache, braun-faltige Kehlstimmsack des Laubfrosches regeneriert nicht nur, wenn an frisch verwandelten, sondern auch, wenn an geschlechtsreifen Tieren operiert. Die braune Verfärbung tritt bei ersteren nur wenig später als an Normaltieren ein, bei letzteren aber ist das Regenerat zunächst geraume Zeit so weiß und glatt wie die übrige Ventralseite. Während des Regenerationsprozesses tritt, abgesehen ron den ersten Wundheilstadien, keine Funktionsunterbrechung ein.

b) Der doppelte Stimmsack des Teichfrosches regeneriert in Form von Hautausstülpungen, die im Vergleich zu den primären kleiner und derber, daher auch weniger durchscheinend sind. Die regenerierten Stimmsäcke werden funktionell nicht in Anspruch genommen.

3) Die Kämme der männlichen Wassermolche sind innerhalb der Laichperiode in hohem Grade regenerationsfähig. Und zwar regenerieren:

a) Die ganzrandigen Kämme des Triton alpestris, marmoratus, vulgaris meridionalis und vulgaris graeca wieder ganzrandig.

b) Der fast ganzrandige (seicht ausgeschweifte) Kamm des Triton blasii (Bastard von Triton marmoratus-Männchen und cristatus-Weibchen) feinkerbig.

c) Der scharf, bisweilen doppelt gesägte Rücken- und der tief ausgeschweifte Schwanzkamm des Triton cristatus durchwegs kerb- 
sägig. War uber der Cloake eine Unterbrechung des primären Kammes, so verläuft der regenerierte Kamm dennoch ununterbrochen. Bei Schwanzamputationen wird der Kamm des Schwanzregenerates zuerst ganzrandig, dann erst kerbsägig.

d) Der buchtig gezähnte Kamm des Triton vulgaris typicus regeneriert ganzrandig.

4) Was die Färbung der Kammregenerate anlangt, so ist

a) diejenige von Triton alpestris zuerst weißlich, fast farb- und gänzlich zeichnungslos;

b) diejenige der übrigen Arten vom Beginn der Regeneration an gefärbt und gezeichnet, wenn auch blasser als normal.

5) Die Hautlappen an der Oberlippe der Tritonmännchen regenerieren, wenn einfach weggeschnitten, noch in der nämlichen, wenn samt dem Kiefer weggeschnitten, trotz früherer Kieferregeneration erst in der nächstfolgenden Brunftperiode.

6) Die ausgebuchteten Hautlappen an den Hinterzehen von Triton rulgaris regenerieren, wenn einfach abgeschnitten, unverändert; wenn mit Zehengliedern oder ganzen Zehen weggeschnitten, ganzrandig; wenn samt dem Hinterbein weggeschnitten, erst in der nächsten Brunftperiode, dann aber wieder ausgebuchtet.

7) Die ausgebuchteten Schwimmbäute zwischen den Hinterzehen von Triton palmatus regenerien:

a) noch während der laufenden Brunftperiode: wenn einfach ausgeschnitten, in ausgebuchteter Gestalt, wie primär; wenn mit Zehen oder Zehengliedern weggeschnitten, mit ganzrandigem Saum;

b) erst in der nächsten Brunftperiode: wenn zugleich das Hinterbein amputiert wurde, dann aber wieder mit ausgebuchtetem Saume.

8) Die Endfäden am Schwanze von Triton palmatus, Boscai, Montandoni, vulgaris meridionalis und vulgaris graeca regenerieren, wenn allein weggeschnitten, bisweilen hypertrophisch und schon nach wenig Tagen; wenn samt dem distalen Schwanzdrittel weggeschnitten, auch noch innerhalb derselben, spätestens aber in der nächstfolgenden Brunftperiode; wenn samt noch größeren Schwanzteilen abgeschnitten, entweder erst in der nächsten Brunftperiode oder gar nicht mehr. Im Falle ihrer Regeneration samt Schwanzteilen gehen die Endfäden, auch solche, die primär von einem abgestutzten Schwanzende ihren Ursprung nahmen (Tr. palmatus), allmählich in den Schwanzsaum über, wie dies z. B. bei Tr. vulgaris meridionalis die Norm darstellt. Im Falle der Nichtregeneration resultiert ein lanzettlich zugespitztes Schwanzende, ohne Fadenbildung. 
9) Die scharlachrote Warze an den Halsseiten des nuptialen Triton pyrrhogaster-Männchens regeneriert, wenn allein weggeschnitten, in verkleinerter Gestalt und braunschwarzer Farbe; wenn mit der umgebenden Halshaut abgetragen, wenigstens in der laufenden Brunftperiode nicht mehr.

10) Das nach Amputation regenerierte Hinterbein des männlichen Triton rusconii erhält den Sporn und die distalwärts von ihm liegenden Unebenheiten an der Außenkante des Unterschenkels in typischer Gestalt wieder.

11) Die blauweiße Schwanzbinde des männlichen Triton cristatus erscheint nach Abpräparierung des betreffenden Hautstreifens erst in der nächstfolgenden Brunftperiode wieder; das unmittelbare Regenerat ist braun gleich der Umgebungsgrundfarbe. Nach Amputation von zwei Drittel des Schwanzes bleibt die braune Farbe bestehen, die helle Binde erscheint trotz Wiedereintrittes der Brunft nicht mehr.

12) Die gelbe Vertebrallinie des weiblichen Triton cristatus erscheint nach Abpräparierung des betreffenden Hautstreifens und Verheilung der Wunde in besonderer Schärfe und Farbensattheit, wie bei jungen, frisch metamorphosierten Exemplaren; sie dunkelt aber im weiteren Verlaufe und verschwindet endlich bis auf geringfügige Reste.

\section{B. Kompensationen.}

Folgende Farbhypotypien der Regenerate bemächtigen sich unverletzt gebliebener Nachbarpartien:

13) Wird aus dem braunen Kehlstimmsack des männlichen Laubfrosches ein Mittelfeld ausgeschnitten, so regeneriert dieses in Weil, welche Farbe auf die stehengebliebenen Ränder übergreift und erst später wiederum einer vom Centrum aus neu auftretenden Bräunung. Platz macht.

14) Die Verbleichung an regenerierten Strecken der Tritonenkämme geht auf die benachbarten, nicht operiert gewesenen Kammstrecken über, besonders bei Triton alpestris, wo die Hypotypie in der Färbung und dem Zeichnungsmangel des Regenerats am schärfsten ausgesprochen ist.

15) Die Verdüsterung an regenerierten Hautstïcken des männlichen Triton cristatus-Schwanzes, die dem Bereiche der blauweißen Flankenbinde angehören, teilt sich nur dem Grenzgebiet der Wunde mit; sie bringt dagegen die gesamte Binde zugunsten der braunen 
Grundfarbe zum Schwund, wenn nicht bloß Hautdefekte, sondern ganze Schwanzteile zu restituieren waren.

Folgende Formhypotypien der Regenerate teilen sich unverletzten Nachbarpartien mit:

16) Wird aus dem faltigen Kehlstimmsack des männlichen Laubfrosches ein Mittelfeld ausgeschnitten, so regeneriert eine glatt" gespannte Haut dariber und auch die Falten auf den stehengebliebenen Rändern verschwinden.

17) Der seicht ausgeschweifte Kamm des Triton blasii wird während und nach der Regeneration benachbarter Kammstrecken im Gegensatz zu diesen, welche gekerbt regenerieren, vollends ganzrandig.

18) Der scharf und tief gesägte Rückenkamm des Triton cristatus erleidet bei Regeneration des Schwanzkammes Abrundung und Verflachung der Zacken, außerdem, wenn er doppelt gesägt war, Einschmelzung der Zacken zweiter Ordnung; der tief ausgeschweifte Schwanzkamm derselben Art wird bei Regeneration des Rückenkammes ganzrandig. Beide Kompensationen sprechen sich auf primär ununterbrochenen Kämmen deutlicher aus als auf solchen, die über der Cloake eine Einsattelung anfwiesen. Bei partiellen Schwanzamputationen wird der Kamm des stehengebliebenen Schwanzteiles zuerst gleich dem des regenerierenden Teiles ganzrandig, dann kleinkerbig; auf dem Rückenkamm in diesem Falle besonders starke Formreduction der Zacken.

19) Der gezähnte Kamm des Triton vulgaris typicus wird während und nach der Regeneration benachbarter Kammstrecken in Übereinstimmung mit den letzteren ganzrandig oder erfährt - bei Regeneration kleinerer oder weit entfernter Strecken (z. B. Nackenkamm bei Regeneration distaler Schwanzkammstrecken) - Verminderung der Zacken und Reduction (Verflachnng und Abrundung) der dann noch iubriggebliebenen.

20) Amputation des ventralen Schwanzsaumes übt auf den Rückenkamm gar keinen EinfluB aus, und umgekehrt; im dorsalen Schwanzsaum werden bei Entfernung des rentralen einige Zacken eingeschmolzen, ebenso im ventralen bei Entfernung des dorsalen. Die kompensatorische Wirkung ist aber in antero-posteriorer Richtung stärker als in dorso-ventraler.

21) Hervorzuheben ist der Unterschied in den Erfolgen von regenerativer und kompensatorischer Regulation: der ausgeschweifte Kamm von Triton blasii wird durch Regeneration gekerbt, durch 
Kompensation ganzrandig; der ausgeschweifte Schwanzsaum von Triton cristatus wird durch Kompensation ganzrandig, durch Regeneration, wenn nur er allein regeneriert, kerbsägig, und erst wenn er zusamt dem Skelet- und Muskelteil des Schwanzes regeneriert, auch ganzrandig.

\section{Andre Ergebnisse.}

22) Durch hohe Temperatur gelingt es, die Geschlechtsreife der Froschlurche, welche nicht bei allen Arten im gleichen Alter, durchschnittlich aber 3 bis 4 Jahre nach der Metamorphose einzutreten pflegt, um beinahe die Hälfte dieser Zeit zu beschleunigen.

23) Operierte Molche zeigen einen stärkeren Geschlechtstrieb als unverletzte und setzen stets früher als diese ihre Geschlechtsprodukte $a b$.

24) Die Brunfterscheinungen und Nuptialattribute der Molche können durch niedrige Temperatur und Luftreichtum des Wassers, am meisten mittels Durchlüftung von reinem Sauerstoff, gesteigert oder hervorgerufen werden.

25) Die männlichen sekundären Sexualcharaktere des Triton rusconii (Sporn und Hechtschnauze) treten ungefähr $1_{1 / 4} \mathrm{Jahr}$ vor Erreichung der Geschlechtsreife auf.

\section{Literaturverzeichnis.}

Barfurth, Dietrich, 1894, Experimentelle Regeneration überschïssiger Gliedmaßenteile (Polydactylie) bei den Amphibien. Arch. f. Entw.-Mech. Bd. I. 1. Heft. S. 91-116. Taf. V.

Bedriaga, Jacques De, 1897, Die Lurchfauna Europas. II. Urodela, Schwanzlurche. Bull. Soc. Imp. Nat. Moscou. XLIV.

Blackwall, J., 1844, 1845, Report on some Researches into the Structure, Function and Oeconomy of the Aranei etc. Brit. Ass. Report. 14. meet. York. p. $62-79$.

Bondage, E., 1897, Sur la régénération tétramerique du tarse des Phasmides. Comptes rendues Acad. Sc. Tome 124. Paris.

Buschkier, Alfred, 1906, Ein Fall von anormal starker Flossenbildung an verschiedenen Fischen im selben Behälter. Wochenschr. f. Aquarien- u. Terrarienkunde. III. Nr. 36 . S. $427-428$.

_- 1907, Zur Frage nach dem Ursprung anormaler Flossenbildungen bei Fischen. Wochenschr. f. Aquarien- u. Terrarienkunde. IV. Nr. 6. S. 66-68.

Brrnes, Esther F., 1904, Regeneration of the Anterior Limbs in the Tadpoles of Frogs. Arch. f. Entw.-Mech. Bd. XriII. 2. Heft. S. 171-177. Taf. X. 8 Fig.

Ćerný, Adolf, 1907, Tersuche ïber Regeneration bei siißwasser- und Nacktschnecken. Arch. f. Entw.-Mech. Bd. XXIII. 4. Heft. S. 503-510. Taf, XXI. 
Driesch, Hans, 1901, Die organischen Regulationen. Vorbemerkungen zu einer Theorie des Lebens. Leipzig, W. Engelmann. S. 65, Fußnote.

Dürigen, Bruno, 1897, Deutschlands Amphibien and Reptilien. Magdeburg, Creutzsche Verlagshandlung.

Fraisse, Paul, 1885, Die Regeneration von Geweben und Organen bei den Wirbeltieren, besonders Reptilien und Amphibien. Cassel u. Berlin, Fischer. S. 152.

Friedrich, Paul, 1906, Regeneration der Beine und Autotomie bei Spinnen. Arch. f. Entw,-Mech. Bd. XX. 1. Heft. S. 38-47. Taf. II.

Giard, Alfred, 1897, Sur les régénérations hypotypiques. C. R. Soc. Biol. Tome IV $(\mathbf{X})$. p. 315-317.

Godelmanx, Robert, 1901, Beiträge zur Kenntnis von Bacillus Rossii Fabr. mit besonderer Beriicksichtigung der bei ihm vorkommenden Antotomie und Regeneration einzelner Gliedmaßen. Arch. f. Entw.-Mech. Bd. XII. 2. Heft. S. $265-301$. Taf. VI.

Herbst, Curt, 1896, 1899, 1901, Über die Regeneration antennenähnlicher Organe an Stelle von Augen. Arch. f. Entw.-Mech. Bd. II, IX n. XIII.

Kammerer, PaUl, 1900, Künstliche Ernährung futterverweigernder Terrarientiere. Natur u. Hans. Bd. VIII. Nr. 13. S. 228-231.

__ 1905, Die angeblichen Ausnahmen von der Regenerationsfähigkeit bei den Amphibien. Centralbl. f. Physiologie. Bd. XIX. 18. Heft. S. $684-687$, in den Verhandl. d. morphol.-physiol. Gesellsch. zu Wien, Sitzung v. 21. Nov.

__ 1907, Bastardierung von Flußbarsch (Perca fluviatilis L.) und Kaulbarsch (Acerina cernua L.). Arch. f. Entw.-Mech. Bd. XXIII. 4. Heft. S. 511-551. 1 Fig. Taf. XXII, XXIII.

Kohlwey, Heinrich, 1897, Arten- und Rassenbildung. Leipzig, W. Engelmann. Konschelt, E., 1907, Regeneration u. Transplantation. Jena, G. Fischer. S. $128_{2}$. Múhelx, LudwiG v., 190万̃, Die herpetologischen Verhältnisse des Mecsekgebirges und der Kapela. Annales Musei Nationalis Hungarici. Bd. III. S. 256-316. Vgl. bes. Fig. 4, 5, 6 u. 9 .

Przibram, Hans, 1905, Quantitative Wachstumstheorie der Regeneration. Centralbl. f. Physiol. Bd. XIX. 18. Heft. S. 682-684, in den Verhandl. morph.physiol. Gesellsch. Wien, Sitzung v. 21. Nov.

SchreIBER, EGID, 1878, Über den Rippenmolch, Pleurodeles Waltlii. Zoolog. Garten. XIX.

Schultz, Eugen, 1898, Über die Regeneration von Spinnenfüßen. Travaux de la Soc. Imp. Natural. St. Pétersbourg. Vol. XXIX.

— 1905, Atavistische Regeneration beim Flußkrebs. Arch. f. Entw.-Mech. Bd. XX. 1. Heft. S. 38-47. Taf. II.

SPallanzani, 1768, Prodromo di un opera ad imprimersi sopra le riproduzioni animali. Modena.

Tornier, Gustav, 1897, Über Schwanzregeneration und Doppelschwänze bei Eidechsen. Sitzungsber. d. Gesellsch. Naturforsch. Freunde in Berlin. S. 59.

Weismans, August, 1899, Tatsachen und Auslegungen in bezug auf Regene ration. Anatom. Anzeiger. Bd. XV. 3. Heft.

WERBER, ISAAK, 1906, Regeneration der Kiefer bei Reptilien und Amphibien Arch. f. Entw.-Mech. Bd. XXII. 1/2. Heft. S. 1-14. Taf. I. II.

Werner, Franz, 1896, Über die Schuppenbekleidung des regenerierten Eidechsenschwanzes. Sitzungsber. d. Kais. Akad. d. Wissensch. Wien, math.-naturw. Klasse. Bd. CY. S. 123.2 Taf. 
Wolterstorff, Willy, 1903, Über Triton blasii de l'Isle und den experimentellen Nachweis seiner Bastardnatur. Zoolog. Jahrbiicher, Abteil. f. Systematik, Geographie u. Biologie d. Tiere. Bd. XIX. 5. Heft. S. 647-661.

Zuelzer, Margarete, 1907, Über den Einfluß der Regeneration auf die Wachstumsgeschwindigkeit von Asellus aquaticus. Arch. f. Entw.-Mech. Bd. XXV. 1/2. Heft.

\section{Erklärung der Abbildungen.}

Die halbschematischen Figuren 1-4 (ausgenommen $4 c$ ) sind nach Skizzen des Herrn Stud. phil. J. H. KLintz ausgeführt, Fig. $4 e$ und 5 von Herrrn Universitätslehrer AdoLF KASPER, Fig. 6-8 von mir selbst entworfen. Fig. $1-5$ in natürlicher Größe, Fig. 6 in vier-, Fig. 7 in 2,5-, Fig. 8 in dreifacher Vergrößerung. Belegmaterial im entwicklungsmechanischen Museum der Biologischen Versuchsanstalt in Wien.

\section{Tafel II.}

Fig. 1. Triton marmoratus o mit normalem Kamm.

Fig. 1 a. Derselbe mit regeneriertem Kamm. 20. V.--7. VIII. 07.

Fig. 2. Tr. marmoratus o $\times T r$. cristatus carnifex \& ( $T r$. blasii «) of mit normalem Kamm.

Fig. $2 a$. Derselbe mit regeneriertem Kamm. 28. II.-7. III. 07.

Fig. 3. Tr. alpestris of mit normalem Kamm.

Fig. $3 a$. Derselbe mit regeneriertem Kamm. 1. III.-30. III. 07.

Fig. 4. Tr. cristatus of mit normalem Kamm und normaler Schwanzflankenbinde.

Fig. $4 a$. Derselbe, Kamm in ganzer Länge regeneriert (1.-6. IV. 07).

Fig. 4 b. Derselbe, nur der Rückenkamm regeneriert, Schwanzkamm kompensatorisch reduziert $(1 .-16$. IV. 07); außerdem Regeneration der lateralen Schwanzhaut mit der Flankenbinde (8. IV. 06-5. IV. 07).

Fig. $4 c$. Derselbe, distale Schwanzhälfte samt Kamm regeneriert, Rückenkamm kompensatorisch reduziert (8. IV.-30. V. 06). Schwanzflankenbinde nicht wiedergekommen selbst bis $8.1 \mathrm{~V} .07$.

\section{Tafel III.}

Fig. 5. Triton vulgaris subsp. typica $\succsim$, mit normalem Kamm.

Fig. 5a $a$. Derselbe, mit regeneriertem Rückenkamm, kompensatorisch reduziertem Schwanzkamm. 20.-25. IV. 06.

Fig. $5 b$. Derselbe, $2 / 3$ Schwanz samt Kamm regeneriert, Riickenkamm kompensatorisch reduziert. 22. IV. 06-5. V. 07.

Fig. 5 c. Derselbe, mit regeneriertem dorsalen Schwanzsaum, kompensatorisch reduziertem Rückenkamm. 20.-28. IV. 06.

Fig. 6. Hinterbein von Tr. vulgaris subsp. typica of zur Brunftzeit, normal.

Fig. $6 a$. Dasselbe nach Regeneration der Phalangen. 26. III.-20. IV. 06.

Fig. 7. Schwanzende von Tr. palmatus of zur Brunftzeit, normal.

Fig. 7 a. Dasselbe, Anfangsstadium der Regeneration nach Amputation der Schwanzhälfte. 8.-30. IV. 05.

Fig. $7 b$. Dasselbe, definitives Stadium der Regeneration, am 5. IV. 07.

Fig. 8. Hinterbein von Triton rusconii, normal.

Fig. $8 a$. Dasselbe regeneriert. 2. VII. 0万-20. III. 06. 


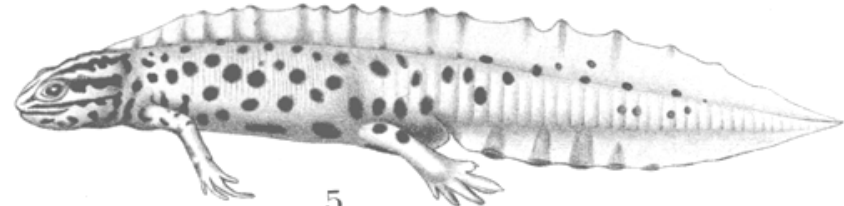

5.

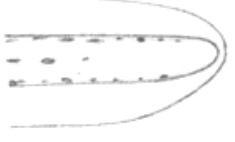

$7 \mathrm{a}$.
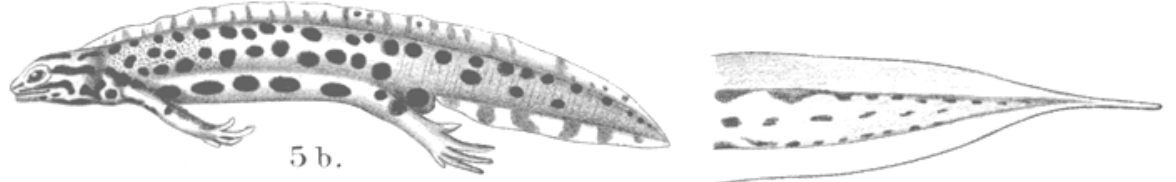

$7 \mathrm{~b}$.
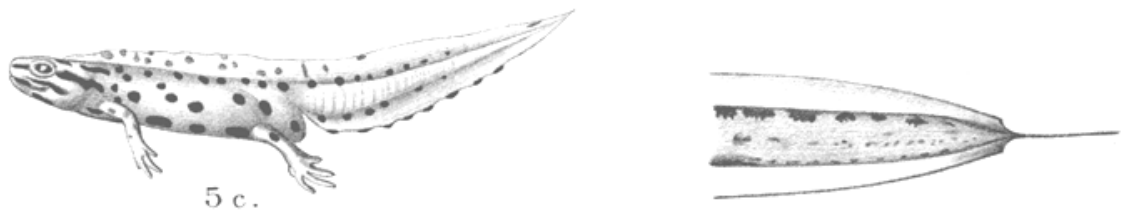

7.
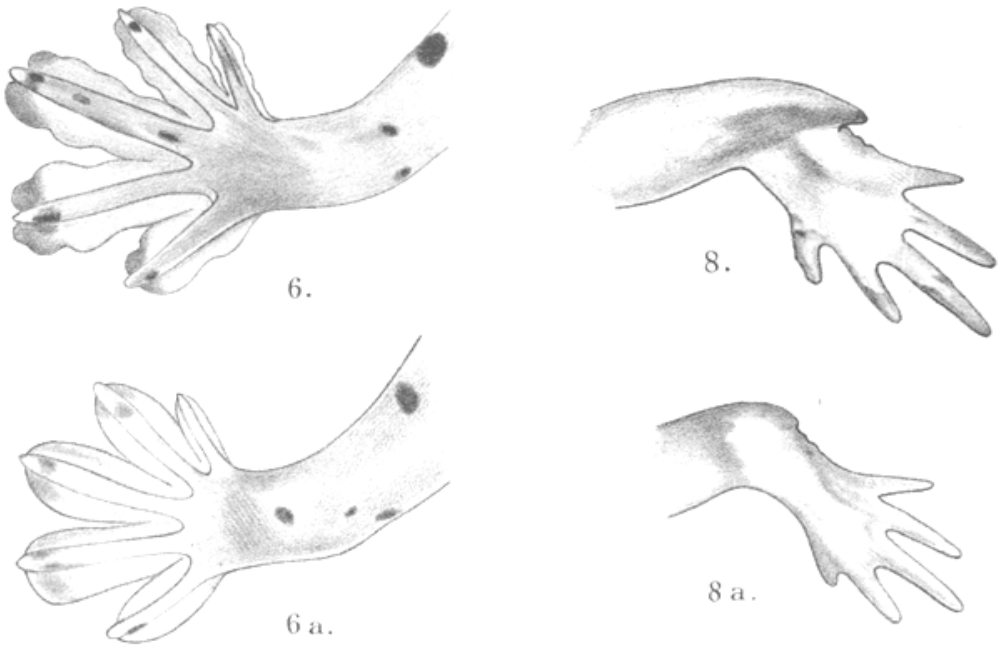